\title{
Va-t-on vers une inversion de la tendance à toujours plus de mobilité ?
}

\author{
Jean-Loup Madre ${ }^{1,}$ Yves D. Bussière ${ }^{2,}$ \\ Roger Collet ${ }^{1}$ et Irving Tapia Villareal ${ }^{1}$
}

\author{
${ }^{1}$ UPE, IFSTTAR, DEST \\ 2 rue de la Butte Verte, \\ 93166 Noisy-le-Grand Cedex, France. \\ Tél: (33) 145925553 \\ jean-loup.madre@ifsttar.fr \\ ${ }^{2}$ Fac. d'Économie, BUAP, \\ Av. San Claudio y 22 Sur s/n, San Manuel, \\ Ciudad Universitaria, \\ 72240 Puebla, Mexique. \\ Tél: (52) 2222843556 \\ ydbussiere@yahoo.ca
}

Rapport soumis pour la Table Ronde de l'International Transport Forum, OCDE, Paris, 29-30 novembre 2012. 


\section{Résumé :}

Dans la plupart des pays industrialisés on assiste depuis le début des années 2000 à une stagnation de la mobilité urbaine et du trafic automobile. En France, le Bilan de la Circulation établi par la Commission des Comptes Transport de la Nation montre une rupture de tendance analogue qui a été confirmée par les Enquêtes Ménages Déplacements (EMD dans la plupart des grandes villes, notamment Lille, Lyon, Strasbourg, puis par l'Enquête Nationale Transport et Déplacements (ENTD) qui permet de l'imputer essentiellement aux habitants des grands pôles urbains et de la resituer dans une vision d'ensemble de la mobilité: les déplacements sont moins fréquents (journée continue) et moins exclusivement automobiles (comportements multi-modaux des jeunes adultes), la motorisation des ménages décroît au cœur de l'agglomération parisienne comme d'ailleurs de celle de Londres...

Ce plafonnement de la circulation traduit-il l'approche de la saturation (découplage entre évolutions des trafics et des revenus dans les régions les plus denses ou au-delà d'un certain niveau de vie?) ou plutôt la superposition d'évolutions contraires (poursuite de la croissance chez les ruraux et péri-urbains vs. recul chez les habitants des zones les plus denses)? S'agit-il d'un phénomène structurel (vieillissement de la population...) ou conjoncturel lié à l'augmentation et à la volatilité du prix des carburants, à la récession ?

Nous examinerons ces questions à la lumière de données collectées en France, complétées par quelques données de pays développés pour ensuite les comparer à quelques villes mexicaines, afin d'envisager dans quelle mesure et à quel horizon ces tendances pourraient s'étendre dans les pays du Sud.

Mots-clefs : Prospective, Mobilité, Motorisation, Rupture de tendance.

\section{Will we see a reversal of the trend of constant increasing urban mobility?}

In most industrialized countries a stagnation of urban mobility and of car traffic is observed since the early 2000s. In France, the Report on traffic by the Traffic Accounts Committee Transport of the Nation shows a similar trend breakdown confirmed the Household Travel Surveys in most large cities, namely Lille, Lyon, Strasbourg, and also by the National Transportation and Travel Survey which shows that this apparent new trend can be attributed mainly to the inhabitants of large urban centers and permits a global overview of mobility: the trips are less frequent (continuous day) and less exclusively by car (multi-modal behavior of young adults), the car ownership decreases in the center of the Paris Region as also in the Centre of London.

Does this leveling off of traffic translate a saturation phenomenon (decoupling growth in traffic and revenue in the densest regions or beyond a certain standard of living ?) or rather the superposition of opposing trends (continued growth in rural and suburban areas vs. decline among residents of the densest areas) ? Is it a structural phenomenon (ageing population ...) or cyclical due to the increase and volatility in fuel prices, recession...?

We will examine these issues in light of data collected in France, completed by additional data of developed countries, to search an answer to these questions and also compare them with case studies on a few Mexican cities to see if these trends could also be present in emergent economies and on what horizon?

Keywords: Prospective Mobility, Motorization, Trend Breakdown. 


\section{Le constat dans les pays développés}

$\mathrm{Au}$ niveau international, après une croissance rapide dans les années '60 et ' 70 , les trafics routiers (en moyenne par tête) ont ralenti et semblent approcher de la saturation dans plusieurs pays industrialisés (Litman (2009); Millard-Ball et Schipper, 2010 ; Newman \& Kenworthy, 2011) - (voir graphique 1). Le bureau australien des infrastructures et de l'économie du transport (BITRE, 2012), qui a rassemblé des séries longues pour 25 pays, explique cette évolution par celles des prix du carburant et de l'activité économique, ainsi que par un effet de saturation lié au temps. Une analyse globale des tendances des 40 prochaines années de la demande mondiale de transport a été présentée par le JTRC/ITF en mai 2011 à Leipzig [OECD/ITF, 2011]. Devant le constat d'une apparente saturation dans les pays développés ce groupe de travail voyait cependant d'un œil critique l'extrapolation de la demande basée sur cette seule hypothèse, faisant ressortir la nécessité de prendre en compte d'autres facteurs comme le renchérissement des carburants et la répartition de la richesse, ainsi que l'importance de l'évolution future de la demande de transport dans les pays émergents. Nos travaux préliminaires sur deux villes mexicaines montrent que le plafonnement de la mobilité pourrait s'y produire vers 2030 .

Dans la plupart des pays développés, la proportion de titulaires du permis de conduire à un âge donné était toujours en progression par rapport aux générations antérieures, et cette augmentation était plus rapide chez les femmes que chez les hommes, rapprochant ainsi leurs comportements. On constate désormais dans une dizaine de pays [Sivak et Schoettle, 2012] un décrochement des taux de permis chez les jeunes, concomitant au développement d'internet, particulièrement sensible pour les hommes ; ces pays sont situés en Amérique du Nord (Canada et USA), là où la diffusion de l'automobile a commencé dès les années 30, dans les pays nordiques (en Norvège et en Suède, mais pas en Finlande), en Europe Occidentale (Grande Bretagne, France et Allemagne, mais ni Suisse, ni Pays-Bas) et dans les pays denses d'Extrême Orient (Japon et Corée du Sud) ; l'essor de la motorisation est trop récent en Europe Centrale (Pologne, Lettonie), et dans une moindre mesure dans les pays méditerranéens (Espagne, Israël), pour voir déjà apparaître un tel phénomène. En France, la baisse de titulaires de permis pourrait être attribuée à la disparition en 1997 du service militaire obligatoire qui permettait aux jeunes hommes un accès quasi-gratuit à la conduite [Avrillier et al., 2010].

Selon une étude comparative auprès des jeunes adultes (20-29 ans) dans six pays industrialisés (Allemagne, Grande Bretagne, France, Japon, Norvège et États-Unis) entre 1975 et 2010, dans la plupart des pays la distance moyenne parcourue a connu un maximum vers la fin des années 90, et au début des années '2000 aux États-Unis, pour ensuite diminuer (graphique 2). Aussi, les jeunes ont moins souvent le permis de conduire et sont moins exclusivement automobilistes que ceux de la génération précédente [Kuhnimohf et al., 2012]. Pour expliquer ce phénomène plusieurs pistes sont proposées : l'accroissement du taux de scolarisation supérieure des jeunes, ce qui retarde leur entrée sur le marché du travail, l'augmentation de l'âge pour former une famille, la hausse du prix du carburant, l'introduction de mesures de gestion de la demande pour diminuer l'usage de l'automobile en ville, et finalement, un changement dans les mentalités. 
Graphique 1 : Tendances internationales véhicules passagers par habitant, 1970-2007

Figure 1 International Vehicle Travel Trends (Litman 2006)

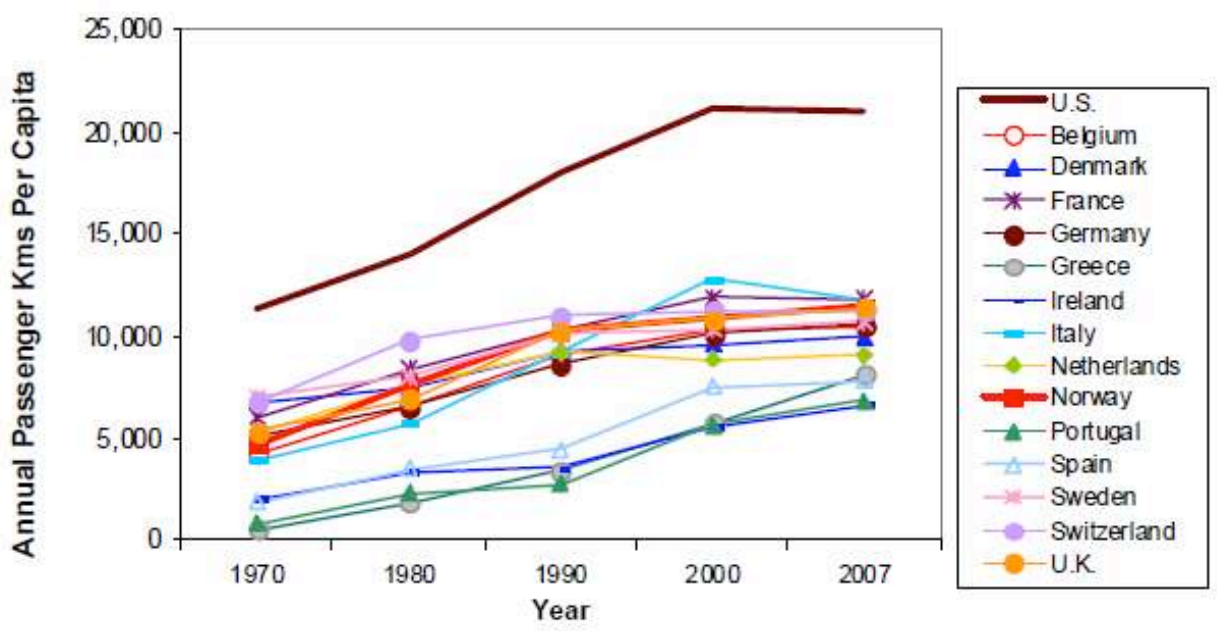

Per capita vehicle travel grew rapidly between 1970 and 1990, but has since leveled off in most $O E C D$ countries, and is much lower in European countries than in the U.S.

Source: Litman, Todd (2009). Evaluation Public Transit Benefits and Costs. Best Practices Guidebook. Victoria Transport Policy Institute. www.vtpi.org.

Graphique 2 : Évolution des véhicules-km (auto-conducteur et auto-passager) par voyageur et par jour pour les jeunes adultes de 20-29 ans (6 pays).

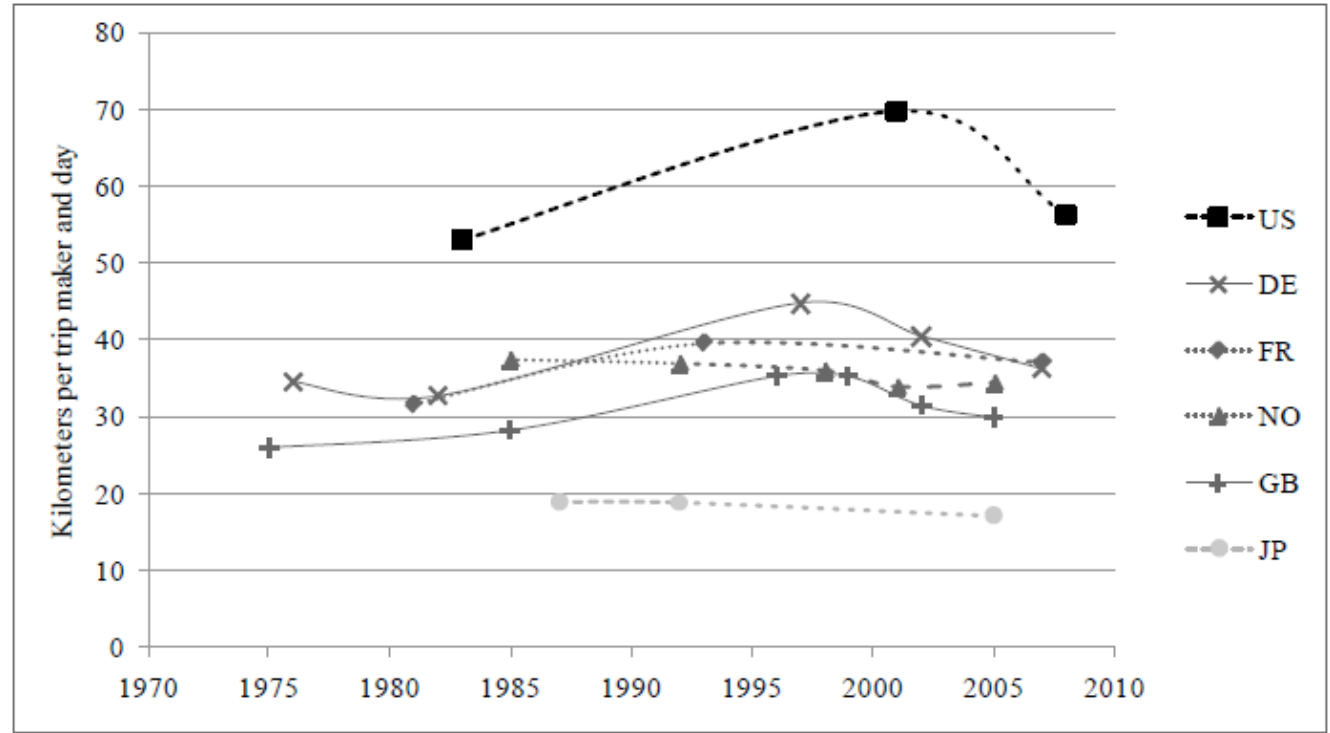

Source : Kuhnimohf et al. (2012).

Pour la Grande-Bretagne, [Metz ,2010] observe que, au cours des 30 dernières années le temps moyen voyagé est resté stable à environ 1 heure par jour (375 heures par personne par année) ainsi que le nombre moyen de déplacements (1000 déplacements par personne par année). La possession d'automobile a plus que doublé ainsi que les vitesses, ce qui avec la hausse des revenus a induit une augmentation importante des distances parcourues, vers une certaine saturation à partir du milieu des années '90 (graphique 3).

Metz avance quelques explications de ce plafonnement de la circulation : moins de déplacements locaux dû à de plus longues absences du domicile [Madre et Armoogum, 1997], l'aggravation de la congestion, une diminution des déplacements liée aux progrès 
dans les télécommunications, ou encore une saturation structurelle de la demande de déplacements.

Graphique 3 : Temps de déplacement (heures par personne par an), distance (milles par personne par an) en Grande-Bretagne

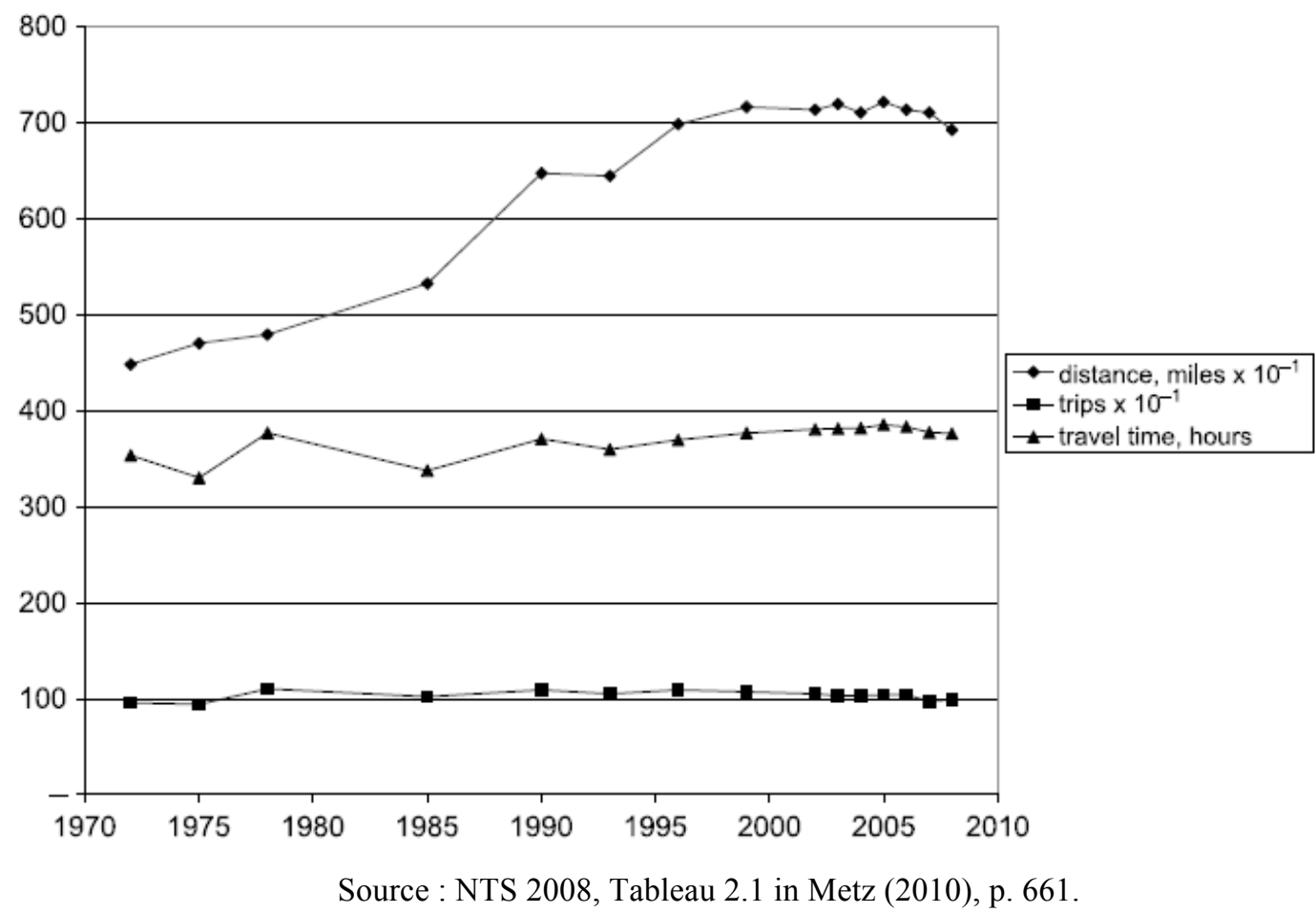

Les tendances observées traduisent-elles l'approche de la saturation via le découplage entre croissance des trafics et des revenus? [Millard-Ball et Schipper, 2010]. Ce découplage se manifeste-t-il d'abord dans les régions les plus denses et/ou au-delà d'un certain niveau de vie ? Ou le plafonnement de la circulation résulte-t-il plutôt de la superposition d'évolutions contraires (poursuite de la croissance chez les ruraux et périurbains vs. recul chez les habitants des zones les plus denses [Goodwin, 20102011]) ? Certains auteurs avancent même l'hypothèse d'une diminution des déplacements à court et moyen terme dans les pays développés, attribuable à divers facteurs socio-économiques [Litman, 2011]. On peut donc se demander s'il s'agit d'un phénomène socio-démographique (vieillissement de la population, redensification du coeur des grandes agglomérations, moins de journées de travail mais plus intensives sans retour chez soi pour déjeuner,...) ou économique lié à l'augmentation et à la volatilité du prix des carburants, à la récession. [Gardes et al., 1996 ; Collet, 2012] ?

L'ERA-MOB (Equipe de Recherche sur la mobilité Associée au laboratoire DEST de l'IFSTTAR) a entrepris l'analyse de ces changements de comportement [Quételard, 2011] constatés par exemple lors des déménagements [Meissonier, 2011]. Ils constituent notamment un enjeu évident en matière de prospective énergétique et environnementale.

\section{Le cas français}

\subsection{Tendances observées}

En France, depuis le milieu des années 2000, le Bilan de la Circulation établi pour la Commission des Comptes Transport de la Nation montre aussi une stagnation des trafics routiers [CCTN, 2012]: la circulation des voitures particulières ne connaît plus de 
progression vraiment significative depuis 2003, c'est-à-dire depuis que le prix du pétrole est clairement orienté à la hausse [SOeS, 2012]. Le seul exemple de croissance plus rapide après 2003 qu'avant s'observe pour les autobus et autocars, témoignant du dynamisme des transports collectifs. Cette rupture par rapport aux tendances antérieures à toujours plus de mobilité a été confirmée par les Enquêtes Ménage Déplacements (EMD) à Lyon, Lille, Rouen, Reims, Rennes, Strasbourg,... [Papon, Hivert ,2008 ; Quételard, 2011], puis par l'Enquête Nationale Transport et Déplacements (ENTD) qui permet de l'imputer essentiellement aux habitants des grands pôles urbains [Hubert, 2009] et de la resituer dans une vision d'ensemble de la mobilité: les déplacements des actifs et des étudiants-élèves sont moins fréquents avec la diffusion de la journée continue [Hubert et al., 2010] et moins exclusivement automobiles. Ainsi, l'augmentation des prix du carburant n'est sans doute pas l'unique facteur ayant infléchi la croissance de la mobilité individuelle ; l'aménagement des programmes d'activité (réduction du temps de travail, télétravail, télé-achat,...) permet d'alléger la charge de transport, rendant son mode réputé le plus pratique, l'automobile, moins indispensable aux citadins. Parallèlement au ralentissement de la circulation automobile individuelle, on assiste également à celui de la motorisation des ménages : elle recule même au cœur des grandes agglomérations, comme dans celles de Londres ou de Paris [STIF, 2012].

D'après les Enquêtes Nationales Transport, c'est seulement dans la tranche d'âge 18-24 ans que l'on observe un repli du taux de permis, particulièrement sensible dans les zones sous influence urbaine (notamment en Île-de-France) (tableau 1) ; il n'apparaît plus dans la tranche 25-35 ans mais il s'est écoulé trop de temps entre les ENT de 1993-94 et 2007-08 pour qu'on puisse affirmer qu'il s'agit d'un rattrapage, puisque la cohorte qui a 18-24 ans en 1994, a 32-38 ans en 2008 [Roux, 2012]. Grâce à des enquêtes moins éloignées dans le temps, nous pouvons constater qu'il n'y a pas de rattrapage à Lille, car le taux de permis des habitants de 25 à 34 ans est aussi en retrait en 2006 par rapport à 1998. Ailleurs, par exemple en Grande Bretagne, la désaffection pour le permis de conduire est attribuée à l'introduction d'une épreuve théorique mi-1996, même si le repli est observable dès 1993 [Noble, 2005]. De même aux États-Unis, on voit comment le recul du permis coïncide avec le durcissement de l'examen dans les différents États [Davis et al., 2012]. 
Tableau 1 : Évolution des taux de permis chez les jeunes adultes selon leur zone de résidence : Île-deFrance (IdF), hors IdF, années 80 à $2000(\mathrm{en} \%)$

\begin{tabular}{|c|c|c|c|c|c|}
\hline \multicolumn{7}{|c|}{ Date } & $\begin{array}{c}\text { Commune de } \\
\text { l'espace à } \\
\text { dominante } \\
\text { rurale } \\
\text { hors Idf } \\
\text { l'espace à } \\
\text { dominante } \\
\text { urbaine } \\
\text { hors Idf }\end{array}$ & $\begin{array}{c}\text { Commune dont : Lille } \\
\text { IdF }\end{array}$ & $\begin{array}{c}\text { France } \\
\text { entière }\end{array}$ \\
\hline Années 80 & 75,7 & 59,9 & 58,7 & 59,7 & 62,3 \\
\hline Années 90 & 69,5 & 69,6 & 64,5 & 56,8 & 66,9 \\
\hline Années 2000 & 78,6 & 67,8 & 54,0 & 48,7 & 65,7 \\
\hline & $\begin{array}{c}\text { Commune de } \\
\text { l'espace à } \\
\text { dominante } \\
\text { rurale } \\
\text { hors Idf }\end{array}$ & $\begin{array}{c}\text { Commune de } \\
\text { l'espace à } \\
\text { dominante } \\
\text { urbaine } \\
\text { hors Idf }\end{array}$ & dont : Lille & IdF & $\begin{array}{c}\text { France } \\
\text { entière }\end{array}$ \\
\hline Années 80 & 87,8 & 81,2 & 81,9 & 78,2 & 81,9 \\
\hline Années 90 & 91,7 & 87,6 & 85,7 & 78,9 & 86,7 \\
\hline Années 2000 & 95,8 & 88,3 & 80,1 & 81,6 & 88,5 \\
\hline
\end{tabular}

Sources: Enquêtes Nationales Transport (ENT) de 1981, 1993 et 2007; Enquêtes Ménages Déplacements de Lille (EMD) de 1987, 1998 et 2006.

\subsection{Perspectives de long terme}

Le ralentissement de l'usage et de l'équipement automobile individuel soulève la question de leurs trajectoires futures. Trois scénarios sont théoriquement envisageables (Goodwin, 2010-2011 ; Collet et al., 2012) :

1) une reprise de la croissance après la pause actuelle,

2) une saturation à des niveaux qu'il faudrait préciser, ou

3) un retournement de tendance après un maximum en plateau, également à préciser.

Quels cadres définir afin de formaliser ces évolutions et de les analyser pour différentes catégories de population et de modes utilisés?

La proposition selon laquelle la motorisation et l'usage automobile individuel pourront croître indéfiniment au fil du temps semble éloignée d'une réalité pratique. Ainsi, s'ils reprennent leur essor au cours des prochaines années, ils finiront par saturer plus tard, pour éventuellement décroître ensuite. En supposant que les ralentissements récemment observés sont bien le signe précurseur du "plafond automobile" (plus connu sous sa dénomination anglo-saxone: "peak car"), nous proposons ici d'en déterminer les niveaux à partir du cas français. Nous excluons donc l'hypothèse 1 , mais nous ne préjugerons pas du caractère permanent ou non du plafond automobile. Autrement dit, nous ne discriminerons pas entre les scénarios 2 et 3 , selon que ce plafond correspond à une saturation de long terme ou à un maximum précédant une diminution.

Malgré une fécondité parmi les plus élevées en Europe, le contexte démographique en France est une croissance faible de la population et un certain vieillissement. En métropole, on prévoit un taux annuel de croissance de $+0.38 \%$ entre 2010 et 2030 . Par 
ailleurs la part des 65 ans et plus qui est passée de $11.4 \%$ en 1950 à $13.9 \%$ en 2000 , à $16.7 \%$ en 2010 , devrait passer à $23.2 \%$ en 2030 et $26.2 \%$ en 2050 (projections INSEE). Dans ce contexte, comment le plafonnement de la circulation des voitures particulières observé depuis les années 2000, surtout dans les zones les plus urbanisées, s'inscrit-il dans le mouvement de long terme de diffusion de l'automobile des couches aisées vers les catégories modestes de la population? Nous décrivons ce processus de 1974 à 2010, France entière et en Île-de-France, en distinguant les ménages de quatre quartiles des niveaux de vie. À chaque date, les ménages moyens des quartiles de la distribution du revenu par unité de consommation sont nommés Q1 à Q4, des plus modestes vers les plus aisés. En remontant aux environs du premier choc pétrolier, nous disposons d'enquêtes annuelles comparables: les Enquêtes INSEE de Conjoncture auprès des Ménages (ECAM) jusqu'en 1994 et le panel Parc-Auto depuis lors. La ré-interrogation du même ménage au moins deux ans de suite par ces panels permet de repérer les changements de comportement et d'améliorer sensiblement la précision pour l'estimation des séries chronologiques.

Afin de situer les niveaux du plafond automobile, nous adoptons une approche par séries temporelles agrégées, en focalisant l'attention sur les catégories de populations susceptibles d'avoir un comportement pionnier: les ménages les plus aisés (moins tenus par la contrainte financière) et les Franciliens (motorisés avant la Province mais contraints par la densité et bénéficiant d'un bon réseau de transports collectifs). La saturation, schématisée par une courbe sigmoïde, apparaît comme une hypothèse médiane entre reprise de la croissance et déclin; en outre, faire dépendre du prix des carburants le niveau de l'asymptote des kilométrages pourrait rendre compte du retournement de tendance observé.

\section{La seconde voiture a pris le relais du premier équipement dans la diffusion de l'automobile}

France entière, le taux d'équipement des ménages les plus modestes a pratiquement rattrapé celui des plus aisés: la proportion de ménages sans voiture dans le premier quartile est tombée de 55\% au milieu des années 70 à 35\% au milieu des années 90 .

C'est la seconde voiture qui a constitué le moteur principal de la réduction des inégalités de motorisation. Le multi-équipement est bien croissant en fonction du niveau de vie jusqu'en 1990. Ensuite, Q2 et Q3 sont proches, et dépassent Q4, les ménages les plus aisés résidant plus souvent dans les zones denses, notamment en Île-de-France (graphique 4).

Comme le taux de multi-équipement ne présente plus d'écarts sensibles en fonction des niveaux de vie, la diffusion sociale de l'automobile atteint ses limites. En Île-de-France, c'est dès le début des années 1980 que les inégalités ont cessé de décroître, et la tendance s'inverse dans la seconde moitié des années 90, avec des inégalités de motorisation désormais un peu plus marquées que France entière.

Au niveau national, les courbes retraçant l'évolution du nombre moyen de voitures par adulte sont croissantes et concaves quel que soit le niveau de vie (graphique 5); la hiérarchie des niveaux de motorisation en fonction des niveaux de vie est respectée sur toute la période étudiée. Il en est de même en Ile-de-France (graphique 6), où la courbe Q1 se stabilise après avoir pratiquement rattrapé Q2 vers le début des années 80 , et où la motorisation des plus aisés n'évolue plus depuis le milieu des années 90. 
Graphique 4: Évolution du multi-équipement des ménages dans chaque quartile du revenu par unité de consommation, France entière

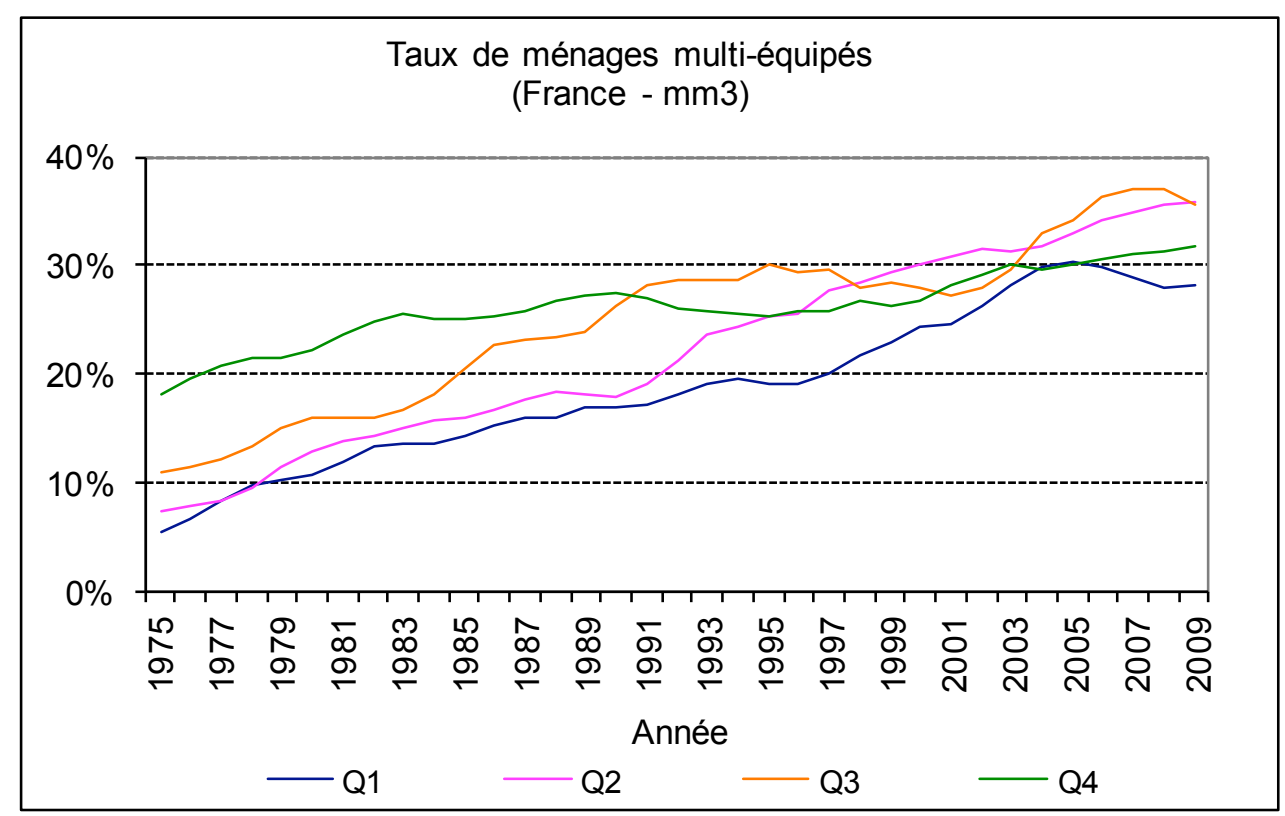

Source : DEST-IFSTTAR $-\mathrm{mm} 3=$ moyenne mobile sur 3 ans

Graphique 5: Évolution du nombre moyen de voitures par adulte dans chaque quartile du revenu par unité de consommation, France entière

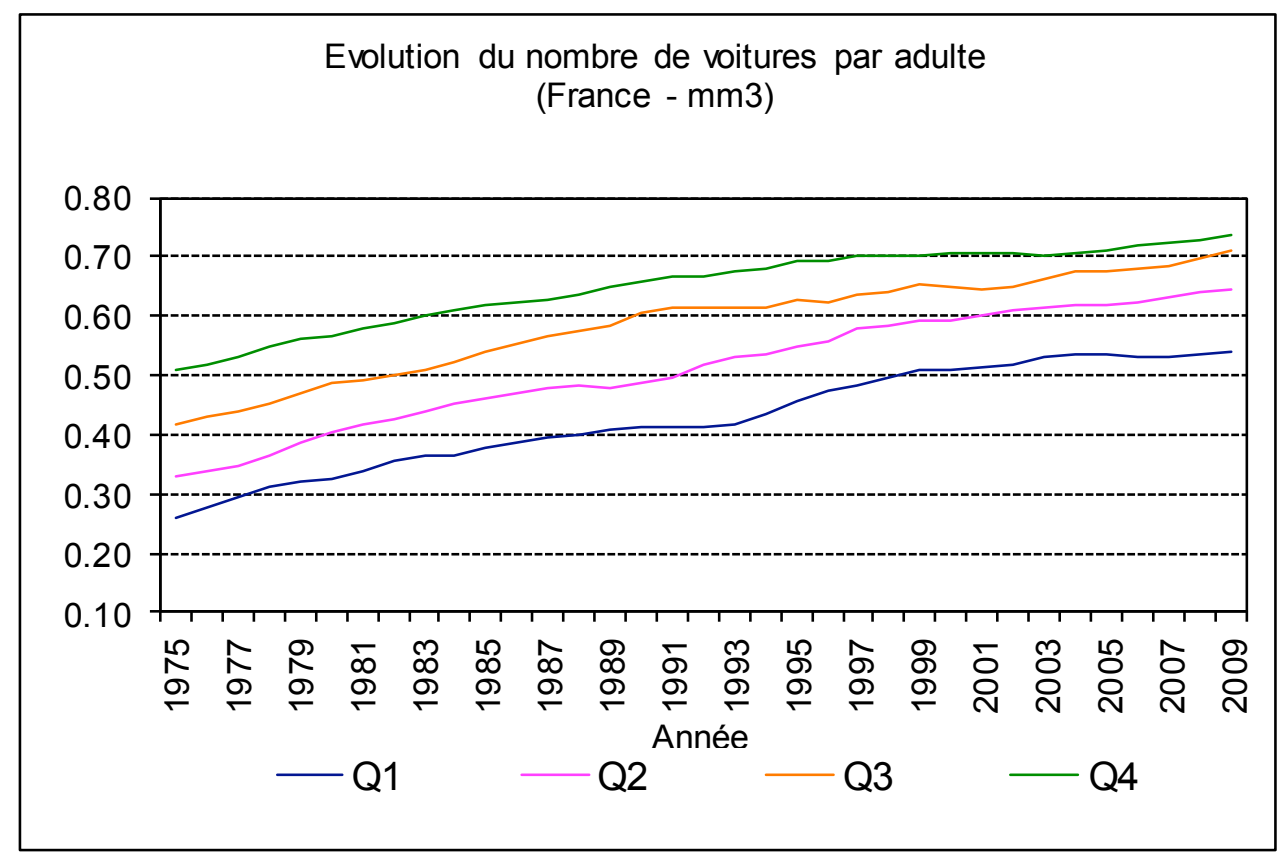

Source : DEST-IFSTTAR $-\mathrm{mm} 3=$ moyenne mobile sur 3 ans 
Graphique 6: Evolution du nombre moyen de voitures par adulte dans chaque quartile du revenu par unité de consommation, Île-de-France

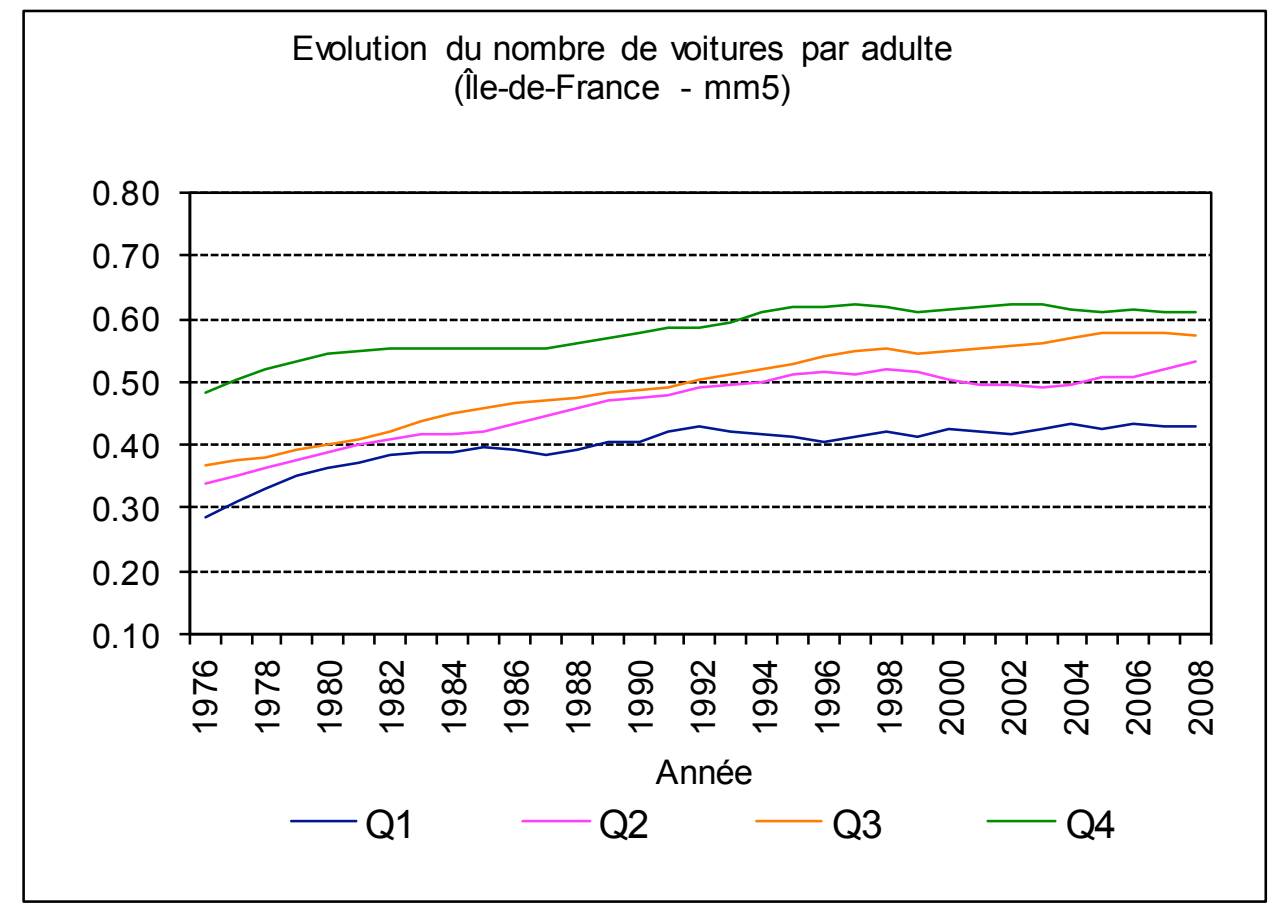

Source : DEST-IFSTTAR $-\mathrm{mm} 5=$ moyenne mobile sur 5 ans.

\section{Une diffusion de l'usage de l'automobile modulée par les fluctuations de prix des carburants}

Après les deux chocs pétroliers des années ' 70 et un plateau dans la première moitié des années ' 80 , le coût d'usage de l'automobile a baissé, d'abord en raison du contre-choc de 1985-86, puis de la diésélisation du parc automobile dans un contexte de moindre taxation du gazole. Globalement, le kilométrage moyen par ménage a augmenté jusqu'en 2000 (graphique 7), puis, puisque la motorisation ne décroît pas, diminué en raison de la baisse du kilométrage moyen par voiture, liée au renchérissement des carburants. En effet, après une pointe observée en 2000, leur prix a continûment augmenté de 2004 à mi-2008, puis connu d'amples fluctuations, établissant un nouveau record au printemps 2012. Les courbes correspondant à Q1, Q2 et Q4 suivent cette forme générale ; celle de Q3 plafonne dès les années 90.

La baisse du kilométrage est plus nette chez les habitants des grandes villes que dans les zones moins denses. Par exemple chez les Franciliens, le plafonnement commence dès le début des années 80 pour Q4, dans la première moitié des années 90 pour Q3, et dans la seconde moitié des années 90, donc avant la flambée de 1999-2000 pour Q2 (graphique 8). Q2, Q3 et Q4 semblent converger en fin de période, alors que Q1 reste en retrait. Lorsque le prix du carburant augmente, les ménages les plus pauvres peinent davantage que les autres à réduire leur usage de la voiture, déjà faible, lorsqu'ils ne disposent pas d'alternatives en transports en commun. 
Graphique 7: Évolution du kilométrage annuel moyen par ménage dans chaque quartile du revenu par unité de consommation, France entière

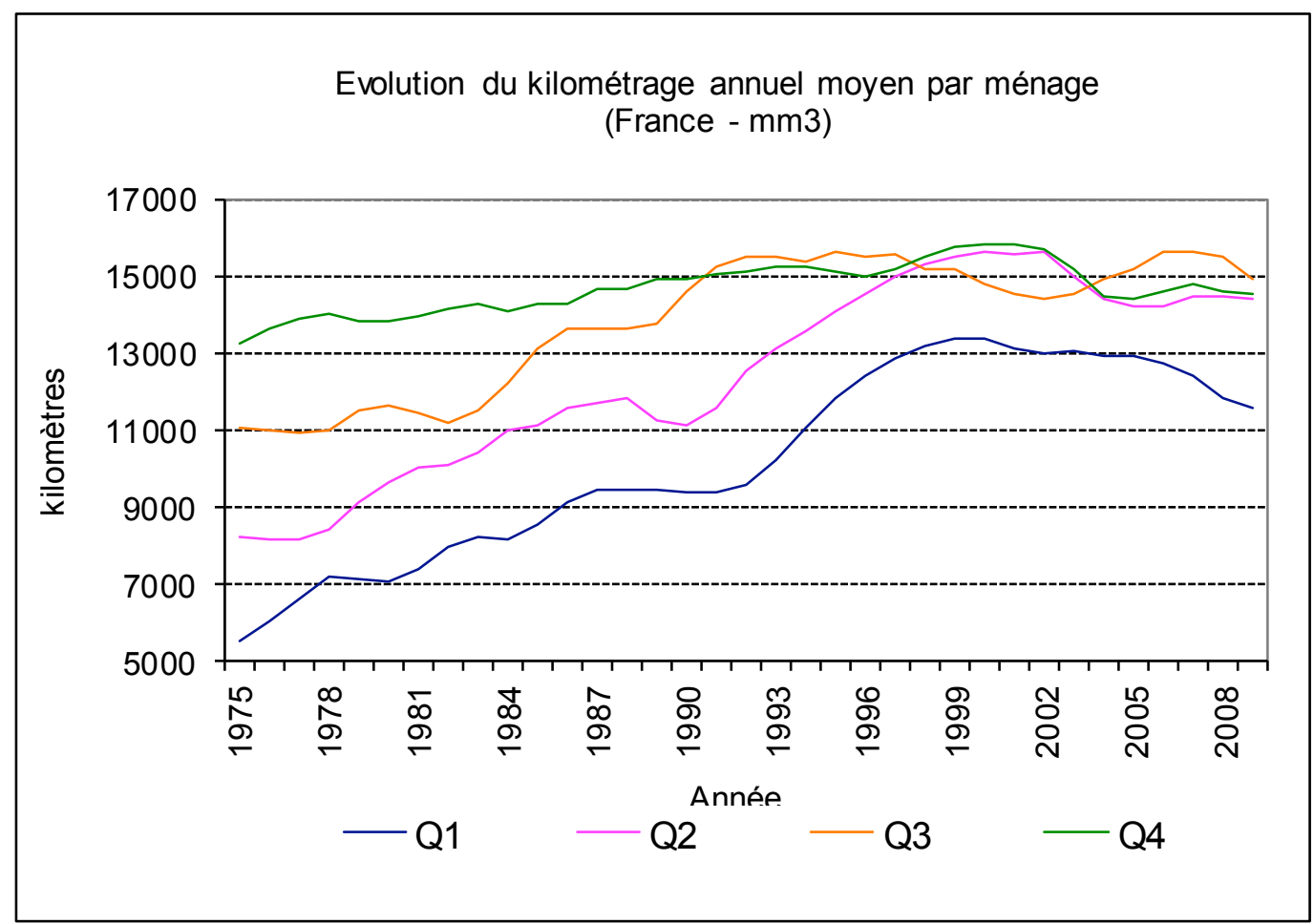

Source : DEST-IFSTTAR $-\mathrm{mm} 3=$ moyenne mobile sur 3 ans.

Graphique 8: Évolution du kilométrage annuel moyen par ménage dans chaque quartile du revenu par unité de consommation, Île-de-France

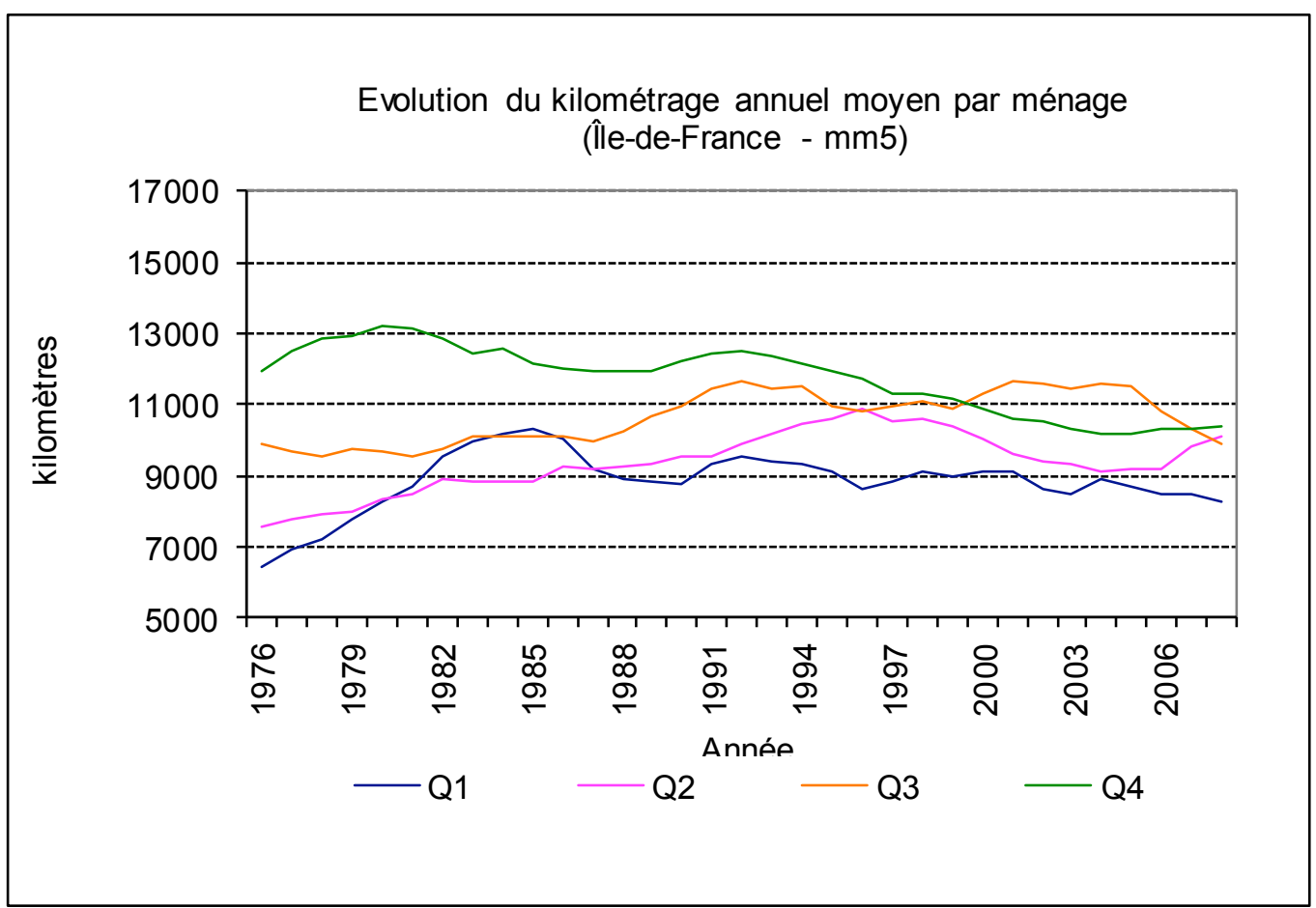

Source : DEST-IFSTTAR $-\mathrm{mm} 5=$ moyenne mobile sur 5 ans. 
Finalement, après une période de statu quo entre le milieu des années 1980 et le milieu des années 1990, la diffusion sociale de l'automobile a repris comme dans les années 1970, particulièrement dans les zones les moins denses où la dépendance à l'automobile est plus forte, notamment pour les ménages à bas revenus [Dupuy, 1999].

\section{Quelles limites à la croissance de la motorisation et de l'usage de l'automobile?}

Théoriquement, la diffusion sociale d'un bien au fil du temps peut être représentée par une courbe sigmoïdale qui tend vers une asymptote : le niveau de saturation. Dans l'hypothèse où les comportements automobiles que nous traitons se conforment à ce schéma, qui concorde bien avec le plafonnement de la circulation observé dans les années 2000, nos données collectées depuis le milieu des années '70 recouvrent essentiellement la partie haute de la sigmoïde. Nous avons ajusté le modèle de Chapman-Richards [Richards, 1979] séparément pour chaque quartile, puis sur l'ensemble des ménages.

Pour la motorisation France entière (tableau 2), les dates d'inflexion s'échelonnent de 1961 pour $\mathrm{Q}_{4}$ à 1972 pour $\mathrm{Q}_{1}$ : elles sont donc bien antérieures au début de nos observations et suggèrent des trajectoires de motorisation différenciées entre quartiles. Par contre, les seuils de saturation ne diffèrent pas significativement : nous avons donc estimé le modèle de motorisation en le contraignant par un plafond d'équipement commun à tous les quartiles. Il est estimé de manière assez précise, son intervalle de confiance à $95 \%$ étant de $[0,74 ; 0,78]$ voiture par adulte.

Tableau 2:Courbes sigmoïdes ajustées sur le nombre moyen de voitures par adulte, France entière.

\begin{tabular}{|c|c|c|c|c|c|c|c|}
\hline $\begin{array}{l}\text { Modèle : } \\
\mathrm{R}^{2}=0.999\end{array}$ & $\begin{array}{l}\text { Chapman-R } \\
\text { Contraint (S } \\
9\end{array}$ & $\begin{array}{l}\text { hards (défin } \\
\text { sommun à to }\end{array}$ & $\begin{array}{l}\text { sur } 1920 \\
\text { us les qu }\end{array}$ & $\begin{array}{l}+ \text { inf } \\
\text { irtiles }\end{array}$ & & & \\
\hline$Y$ & Coefficient & Écart type & Student & $P>t$ & [95\% Conf. & Interval] & $\begin{array}{c}\text { Année } \\
\text { d'inflexion }\end{array}$ \\
\hline $\bar{S}$ & 0.7618565 & 0.009165 & 83.13 & 0 & 0.7437356 & 0.7799774 & \\
\hline Quartile1 & & & & & & & 1973 \\
\hline b1 & 0.0328789 & 0.0011699 & 28.11 & 0 & 0.0305659 & 0.0351919 & \\
\hline b3 & 0.825462 & 0.0128132 & 64.42 & 0 & 0.800128 & 0.850796 & \\
\hline Quartile2 & & & & & & & 1970 \\
\hline b1 & 0.0461801 & 0.0018558 & 24.88 & 0 & 0.0425109 & 0.0498494 & \\
\hline b3 & 0.9015596 & 0.0103673 & 86.96 & 0 & 0.8810616 & 0.9220575 & \\
\hline Quartile3 & & & & & & & 1965 \\
\hline b1 & 0.0552784 & 0.0030402 & 18.18 & 0 & 0.0492673 & 0.0612895 & \\
\hline b3 & 0.9188615 & 0.0134812 & 68.16 & 0 & 0.8922069 & 0.9455161 & \\
\hline Quartile4 & & & & & & & 1961 \\
\hline b1 & 0.0654347 & 0.0055028 & 11.89 & 0 & 0.0545548 & 0.0763147 & \\
\hline b3 & 0.9312386 & 0.020351 & 45.76 & 0 & 0.891001 & 0.9714762 & \\
\hline
\end{tabular}

En Île-de-France, les plafonds d'équipement dépendent significativement du niveau de vie des ménages : l'intervalle de confiance à $95 \%$ pour le quartile le plus modeste est $[0,41 ; 0,43]$ voiture par adulte, $[0,52 ; 0,57]$ pour le second quartile, et $[0,59 ; 0,69]$ pour le troisième. Toutefois, on n'observe pas de différence significative entre ce dernier 
et le quartile le plus aisé, dont le plafond d'équipement est estimé entre 0,61 et 0,65 voiture par adulte. En raison de la forte densité en région parisienne, les seuils d'équipement estimés chez les Franciliens sont tous significativement inférieurs à celui précédemment calculé France entière ( 0,76 voiture par adulte).

Pour les kilométrages annuels par ménage, la date d'inflexion est toujours d'autant plus récente que les ménages ont un bas niveau de vie, celles-là s'échelonnant de 1952 à 1974 (tableau 3).

Comme cette hypothèse est acceptable au vu des données, nous estimons un modèle contraint par un plafond $S_{2}$ et par une élasticité fi communs. Le plafond d'usage au prix des carburants en 2010 est alors estimé à $16200 \mathrm{~km} / \mathrm{an}$. On calcule ainsi que le plafond d'usage par ménage en France diminuerait d'environ $2400 \mathrm{~km}$ si le prix des carburants doublait par rapport à son niveau de 2010, passant alors à $13800 \mathrm{~km} / \mathrm{an}$.

Tableau 3: Courbes sigmoïdes ajustées sur le kilométrage annuel par ménage en fonction du prix des carburants, France entière

\begin{tabular}{|c|c|c|c|c|c|c|c|}
\hline Modèle : & Chapman-Richards (défini sur 1920; +inf ) \\
Contraint (S et b4 communs à tous les quartiles) \\
$\mathrm{R}^{2}=0.99704$ \\
\hline
\end{tabular}

En région parisienne et contrairement aux résultats France entière, les plafonds d'usage de l'automobile sont estimés de manière assez précise pour chaque quartile de la distribution des niveaux de vie. Pour un prix relatif des carburants équivalent à celui de 2010, le kilométrage du quartile francilien le plus modeste devrait culminer autour de $8600 \mathrm{~km} / \mathrm{an}, 10000$ pour le second quartile, et 10700 pour les deux quartiles supérieurs. Les intervalles de confiance autour de ces estimations sont relativement réduits et suggèrent des différences significatives entre les trois premiers quartiles. L'élasticité au prix des carburants n'est pas significativement différente d'un quartile à l'autre; le modèle contraint l'estime à $-0,31$.

L'ajustement de courbes sigmoïdes permet de situer la saturation vers 0,76 voiture par adulte, et $16400 \mathrm{~km}$ par an et par ménage ; ce plafond des kilométrages est modulé par une élasticité de $-0,24$ rendant compte des réactions aux évolutions heurtées des prix des 
carburants. Toutefois en Île-de-France, où la congestion de la circulation est forte et le réseau de transports collectifs bien développé, le plafonnement des kilométrages a commencé d'autant plus tôt que les ménages sont plus aisés, avant le renchérissement du carburant des années 2000, laissant penser qu'il s'agit d'un mouvement de fond dont les Franciliens seraient précurseurs.

\section{Changements de comportement ou effet de structure? Une approche démographique}

Depuis le milieu du 20ème siècle, l'usage de la voiture en ville comme ailleurs ne faisait que croître à un rythme soutenu et régulier et aucun ralentissement de cette croissance n'était perceptible. Mais à partir de 2006, toutes les EMD montrent une stabilisation et souvent une baisse de l'usage de la voiture, dont l'évolution de la structure de la population (notamment son vieillissement) n'explique qu'une faible part [Quételard, 2011] :

- à Lille, les évolutions dans la structure de la population auraient dû entraîner une légère croissance de la mobilité (4,02 déplacements par personne et par jour en 2006 contre 4,00 en 1998), mais on en a observé 3,76;

- à Lyon, les évolutions de structure auraient dû entraîner effectivement une baisse de la mobilité mais la baisse observée est bien plus forte, de 3,63 en 1995 à 3,36 en 2006 (3,60 à structure constante) ;

- à Strasbourg, "10\% de la baisse de la mobilité des habitants de la Communauté Urbaine (CUS) est exclusivement lié au phénomène de vieillissement démographique" et "l'augmentation de la part des actifs, scolaires et étudiants pratiquant la journée continue explique mécaniquement $20 \%$ de la baisse de la mobilité" [ADEUS, 2010].

S'agit-il de changements de comportements liés à la conjoncture ou d'effets de structure ? Pour tenter de répondre à cette question nous avons analysé le cas de la Communauté Urbaine de Lille, où ont été réalisées des enquêtes-ménages-déplacements en 1987, 1998 et 2006, en ajustant un modèle de projection démographique ÂgeCohorte permettant d'isoler des effets structurels d'âge et de génération liés au cycle de vie.

Nous avons appliqué à Lille le modèle Âge-Cohorte (modèle décrit dans: Bussière, Madre, Armoogum, 1996), sur différentes variables de mobilité pour la période 20002030. Une première conclusion est que, puisqu'on se déplace moins au-delà d'un certain âge, le vieillissement de la population a un impact certain mais qu'il ne rend compte que partiellement du plafonnement de la mobilité observée dans les années 2000. Le vieillissement, qui donne moins de poids aux jeunes, amplifie le phénomène de plafonnement.

La calibration du modèle nécessite au moins deux années observations assez distantes l'une de l'autre. À partir des trois années d'enquête disponibles $(1987,1998,2006)$ nous avons successivement calibré le modèle avec les 3 années, avec 1987-1998 et avec 1998-2006. Chacune des trois estimations donne des résultats très différents, puisque le plafonnement de la mobilité n'a été constaté que lors de la dernière enquête. Plus récentes les données de calibration, plus grand est le plafonnement. Si l'on se base sur les 2 dernières calibrations, nous obtenons les résultats suivants. À l'horizon 2030, la proportion d'adultes titulaires du permis de conduire devrait stagner de même que les variables suivantes : $\mathrm{km}$ voyagés par personne en automobile individuelle conducteur (graphique 9) ou passager, et une augmentation sensible du nombre de km par jour par 
personne en transports collectifs (graphique 10). Par exemple, le modèle montre au-delà de la renaissance des transports en commun observée dans les années 2000, que la croissance de leur utilisation entre 2000 et 2030 pourrait aller de $+15 \%$ d'après le calage 1987-1998, à $+47 \%$ en calant le modèle sur les deux dernières enquêtes. Les différences significatives observées entre les comportements prévalant jusqu'aux années 1990 et dans les années 2000 expliquent bien le retournement de tendance observé, et conduisent à des projections très divergentes ce qui pour une prospective de long terme soulève à nouveau la question de savoir si les tendances récentes sont structurelles ou conjoncturelles.

Graphique 9: Simulation modèle Âge-Cohorte, Lille 2000-2030 Distance moyenne voyagée par jour par personne en $\mathrm{km}$, auto-conducteur

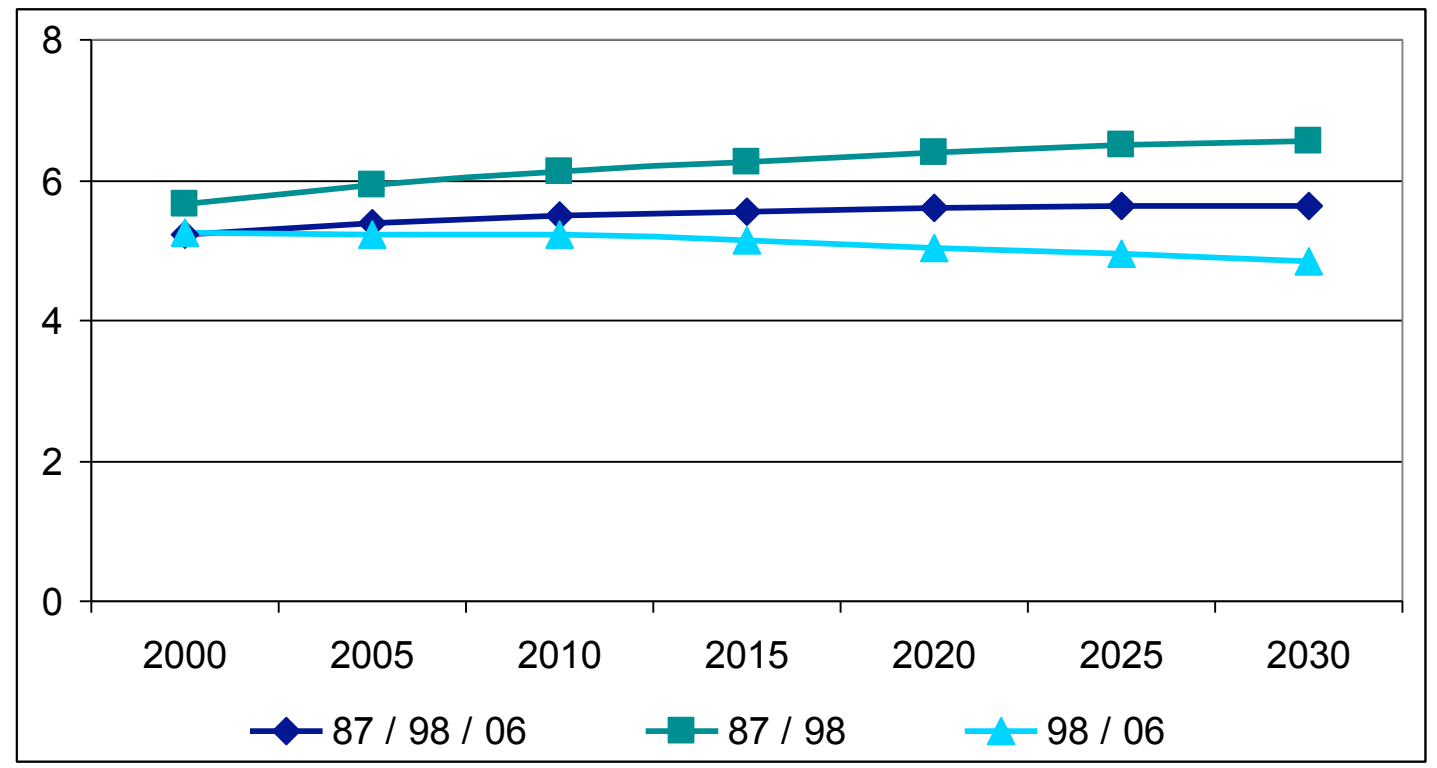

Graphique 10: Simulation modèle Âge-Cohorte, Lille 2000-2030 Distance moyenne voyagée par jour en $\mathrm{km}$, transports collectifs

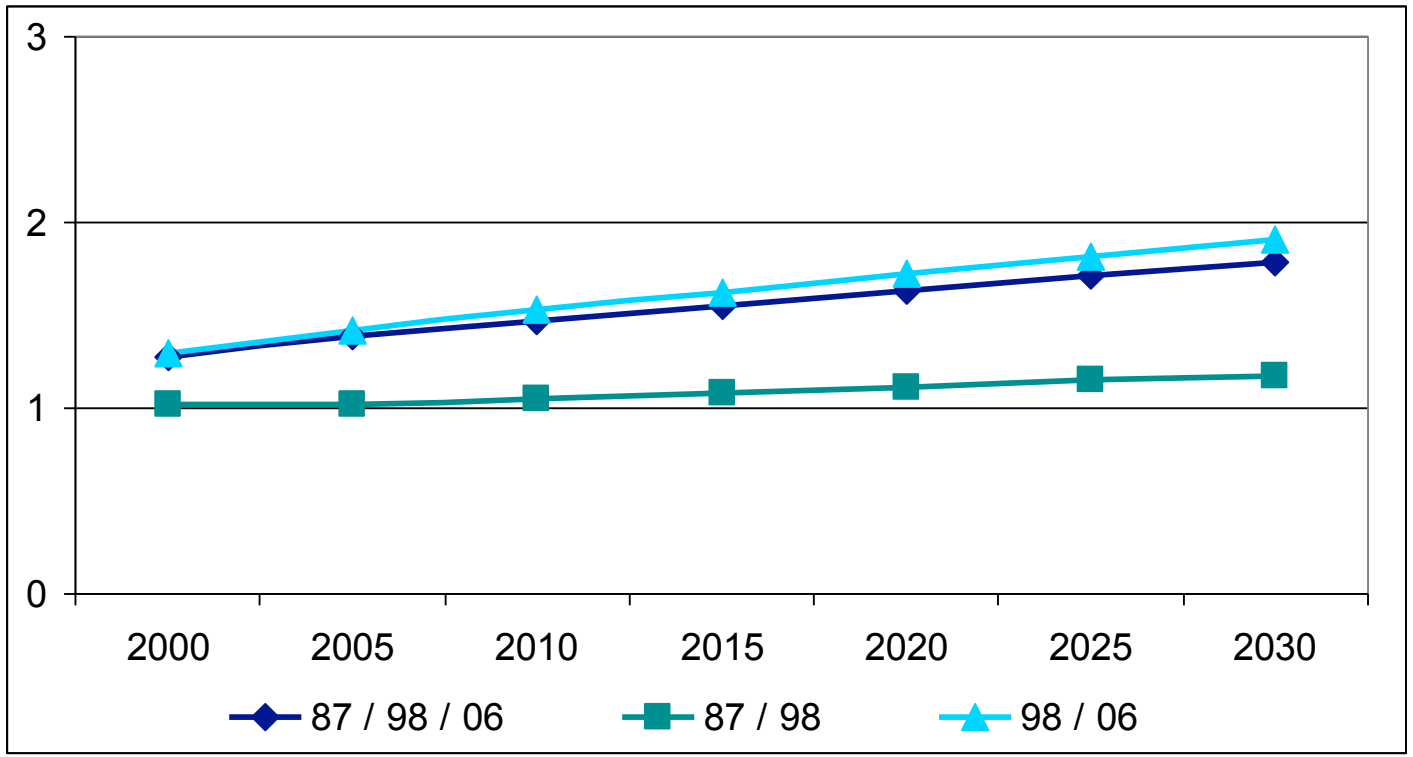




\section{Facteur du vieillissement dans les pays développés vs. dans les pays émergents - exemple du Mexique}

Une première approche simple pour mesurer l'impact de l'évolution de la pyramide des âges sur la mobilité est d'appliquer des comportements de transport stables à une pyramide des âges en évolution sur le long terme. Nous montrerons ici une telle évolution à partir d'une comparaison entre Montréal et Puebla. Le lien entre l'âge ou le cycle de vie, la mobilité et le choix modal sont bien établis et relativement stables dans le temps. Comme on peut l'observer dans les graphiques ci-dessous, la mobilité générale est en forme de cloche. À Montréal, en 1993, elle atteint un sommet vers 35 ans et diminue par la suite régulièrement jusqu'aux âges avancés. À Puebla, pour la même année (1993-4) on observe une courbe quasiment identique mais avec un niveau global de mobilité plus faible. Côté choix modal la mobillité auto-conducteur est en forme de cloche, atteignant un sommet à Montréal vers 40 ans. La forme de la courbe est semblable à Puebla avec un sommet un peu plus tardif, vers 45 ans avec un niveau cependant plus faible. Le mode transport collectif étant en concurrence direct avec l'automobile, la courbe observée est en forme de U dans le cas de Montréal, et adopte une forme similaire dans le cas de Puebla (voir graphiques 11 à 13). Le résultat croisé de ces évolutions donnera inévitablement un usage de l'auto individuelle élevé dans les groupes d'âge actifs, qui se traduit en véhicules-km comme on peut le constater aux États-Unis pour la période 1995-2001-2009 où l'on observe une diminution des véhicules-km chez les plus jeunes avec cependant une augmentation dans les groupes d'âge de plus de 65 ans (graphique 14).

En l'absence de données d'enquête sur une période longue nous avons supposé les comportements de mobilité stables et appliqué ces comportements par groupes d'âge quinquennaux sur une longue période à partir du cas de Montréal et de Puebla. Dans le cas de Montréal nous avons retrouvé un exercice que nous avions fait il y a 22 ans mais qui à l'époque laissait sceptiques les ingénieurs de transport. Nous avons appliqué les comportements de 1982 sur une période de 40 ans (1971-2011) (graphique 15) (Bussière, 1993). Faute de données démographiques détaillées sur une série longue pour la ville de Puebla, nous avons refait le même exercice avec des données de population du Mexique en appliquant les comportements de 1993-4 de Puebla sur une période de 100 ans (1950-2050) (graphique 16). Pour valider la méthodologie nous avons fait une autre simulation sur les données démographiques de Puebla 2000-2050, qui donne des résultats similaires.

Que peut-on en conclure ? Ces simulations ne sont évidemment pas des projections de la demande passée et future car elles ne tiennent pas compte des changements de comportements liés au mode de vie et à la forme urbaine. Elles permettent cependant d'isoler le seul facteur démographique. Dans le cas de Montréal où le baby-boom a été très fort et suivi d'une chute de la natalité on a assisté entre les années ' 70 jusqu'à la fin des années '90 à une augmentation exponentielle des pressions vers la motorisation individuelle et à une baisse des transports en commun, avec pour la motorisation individuelle, un point d'inflexion en 2001 suivi d'une diminution. Exactement la tendance observée dans la grande majorité des villes développées (graphique 1). Sans préjuger des autres facteurs (forme urbaine, accès à l'automobile, style de vie) on peut donc conclure que l'impact démographique dans l'évolution de la motorisation individuelle est important et que nous assistons actuellement à un effet de saturation de cet impact. 
Graphiques 11: Mobilité globale par âge, Montréal et Puebla (1993)

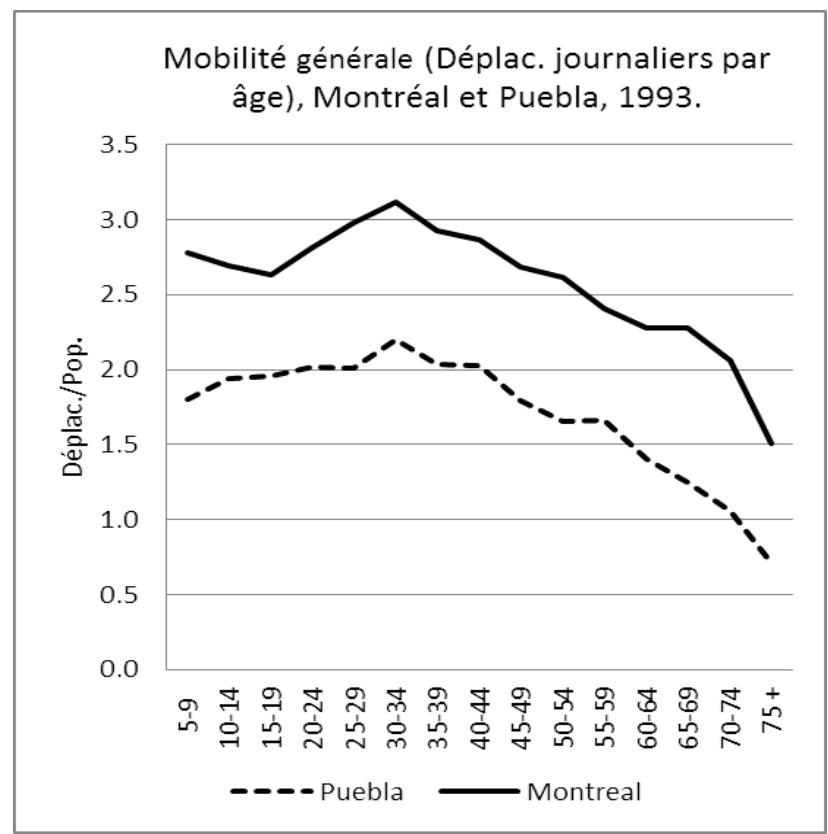

Source : Enquêtes Ménages Origine-Destination.

Graphique 12 : Choix de mode par âge - Auto -conducteur et Transports en commun, Montréal (1982-1987)

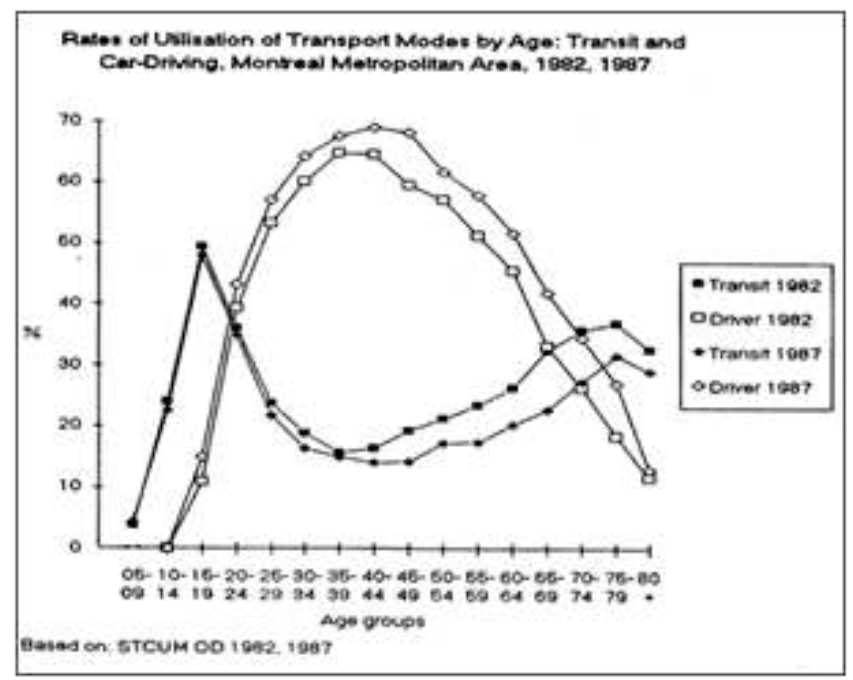

Source : Enquêtes Ménages Origine-Destination.
Graphique 13 : Choix de mode par âge Auto -conducteur et Transports en commun, Puebla (1993)

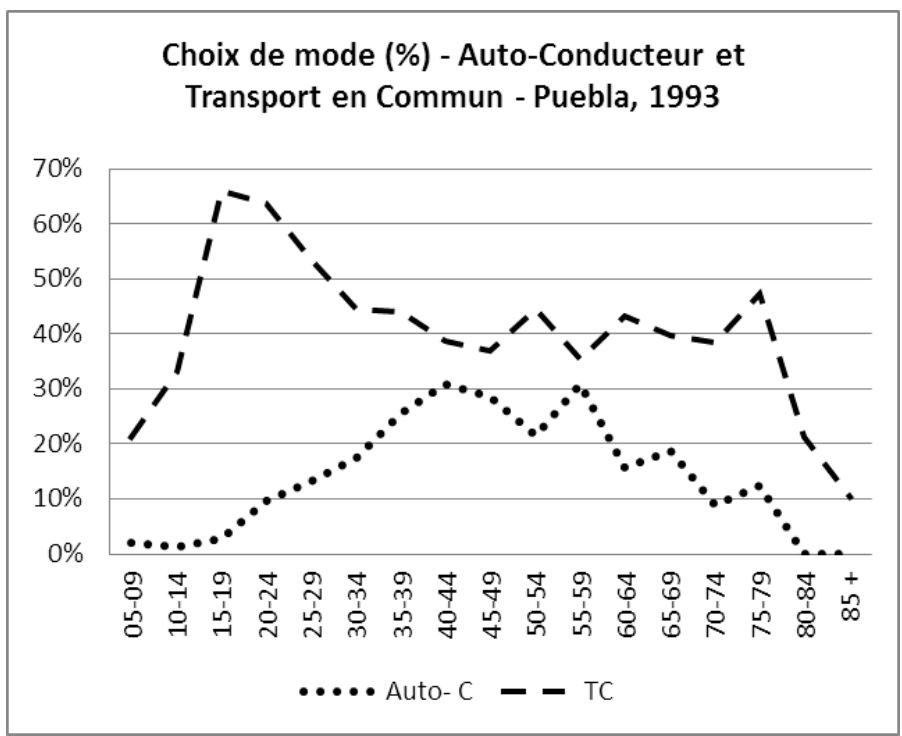

Source : Enquêtes Ménages Origine-Destination.

Graphique 14: Véhicules miles annuels par conducteur par âge, USA, 1995, 2001, 2009

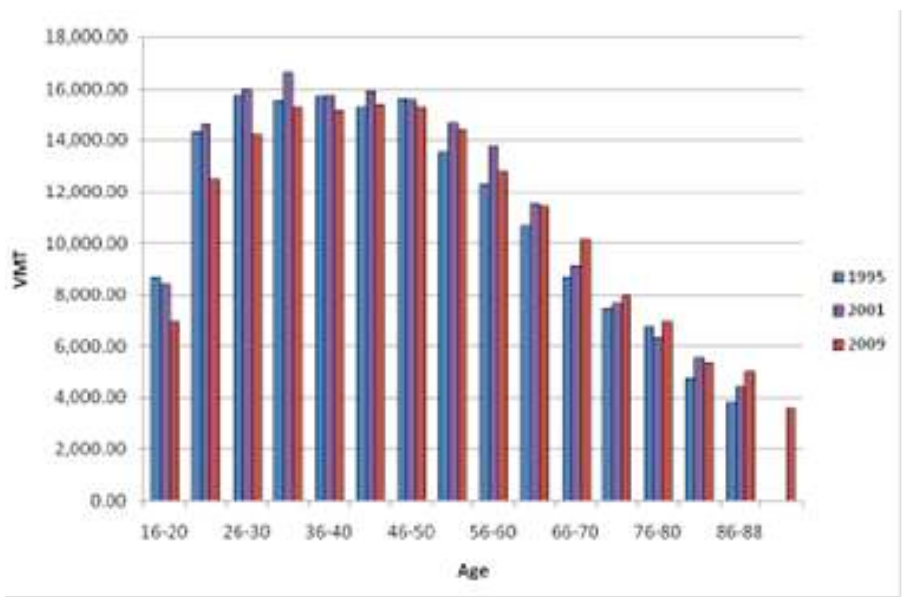

Source: OECD/ITF (2011., p. 29, pdf, www. 
Dans les pays émergents la population est encore jeune mais le phénomène du vieillissement viendra aussi. Au Mexique la croissance de la population qui a été très rapide jusque récemment (taux moyen de croissance de $1,58 \%$ entre 1990 et 2010) devrait ralentir. On prévoit un taux annuel de croissance annuel de l'ordre de $0,67 \%$ entre 2010 et 2030 et d'un rapide et fort vieillissement (proportion de 65 et plus de $3,4 \%$ en 1950 , de $4,2 \%$ en 1990 , de $6,4 \%$ en $2010,12,5 \%$ en 2030 et $22,0 \%$ en 2050 ) Projections CONAPO. Dans les villes la croissance serait un peu plus rapide à cause de la poursuite de l'exode rural. Une simulation sur l'agglomération de Puebla nous indique un commencement de pressions démographiques favorables à la motorisation individuelle vers l'an 2000 au moment même où dans les villes développées on a observé une décélération. Viennent ensuite une trentaine d'années de fortes pressions démographiques favorables à la motorisation individuelle et une décélération pour les transports collectifs. Le point d'inflexion se situe vers 2035 après lequel on assisterait à une décélération de l'auto-conducteur et à une quasi-stabilisation pour les transports collectifs. Qu'en conclure ? Cette simulation représente un scénario minimaliste car il suppose le maintien d'une motorisation faible. Si l'on y ajoutait un facteur croissance du niveau de vie et de motorisation des ménages, le résultat serait explosif pour au moins encore une vingtaine d'années. Une enquête Transport Ménages O-D récente à Puebla (2011) nous révèle des éléments intéressants pour compléter le portrait. Entre 1994 et 2011 la mobilité par habitant est restée stable (1,75 déplacement par jour par habitant) ainsi que la motorisation individuelle qui a suivi la croissance de la population $(+50 \%)$, mais où la persistance de la pauvreté n'a pas permis une augmentation dans la motorisation des ménages. Au contraire, la proportion de ménages équipés est tombée à $33 \%$ en 2011 par rapport à 39\% en 1994 et la proportion de ménages multi-équipés était seulement de 3,8\% en 2011 par rapport à 10,3\% en 1994. Cette évolution reflète en grande partie le maintien de la pauvreté. Si en 1994 19,0\% des ménages souffraient de pauvreté alimentaire le taux n'a pas changé en 2008 avec 19,5\% (graphique 17) (Coneval, 2009). Par ailleurs durant la même période l'âge moyen du parc automobile est passé de 9,4 à 13,0 ans. Une enquête de 2012 dans la ville de Colima sur la côte ouest du Mexique indique un âge moyen du parc auto des ménages de 13 ans. En France on a observé aussi un phénomène de vieillissement du parc auto dont 1'âge moyen est passé de 6,2 ans en 1993 à 8,2 ans en 2007 (Kolli, 2012) mais pour des raisons bien différentes : la diffusion de la deuxième auto qui circule moins et dure plus longtemps.

Pour compléter cette première analyse avec une méthodologie plus complexe qui tient compte de davantage de facteurs nous avons appliqué le modèle Âge-Cohorte sur deux villes mexicaines pour l'ensemble des déplacements. Pour Puebla, sur la période 1996-2030 à partir des enquêtes de 1994 et 2011 (graphique 18) ; pour Cd. Juarez, sur la période 1996-2030 à partir des enquêtes de 1996 et 2006 (graphique 19). Contrairement à nos premières simulations purement démographiques, ces simulations tiennent compte de l'évolution des comportements par groupes d'âges fins qui incorporent indirectement une série de facteurs comme l'évolution de la forme urbaine, le revenu, les modes de vie. Dans le cas de Puebla on obtient un point d'inflexion en 2020 et une diminution par la suite. Dans le cas de Cd. Juarez basé sur un scénario croissance démographique ralentie compte tenu du contexte de violence actuel (taux de croissance annuels moyens projetés de $1,60 \%$ de 2006 à 2012 et de $1,06 \%$ de 2015 à 2030), le point d'inflexion apparaît plus tôt, en 2015, ce qui ne pourrait s'expliquer par le vieillissement avec seulement 5,4\% de personnes âgées de 65 ans et plus en 2015 mais vraisemblablement par un début de saturation de la motorisation individuelle où $72 \%$ des ménages sont équipés comparativement à $84 \%$ en France en $2007-8$ et $36,4 \%$ de ménages sont multi-équipés comparativement à 38\% en France en 2008 (Kolli, 2012). Là aussi l'âge du parc est vieillissant, passant de 11,7 ans en 1996 à 13,8 en 2006, possiblement lié au multi-équipement, au maintien d'une pauvreté relative et à la proximité de la frontière américaine facilitant l'importation peu taxée sur les véhicules de 10 ans et plus 
Graphique 15: Évolution de la demande de transport (Tous modes, Transport en Commun, Auto-Conducteur), Montréal 1971-2011 (1971=1)

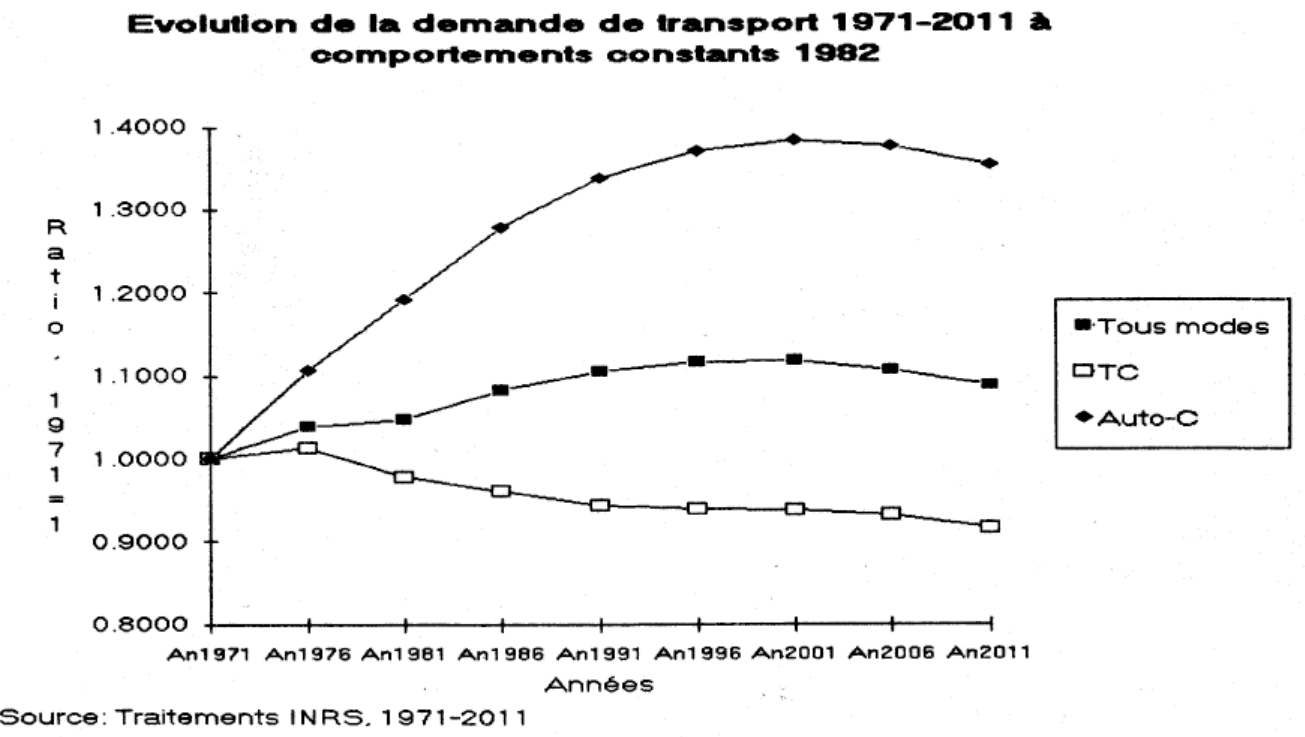

Source : Bussière \& Fortin,1990.

Graphique 16: Estimation de la mobilité par modes, Région métropolitaine de Puebla, 1950-2050.

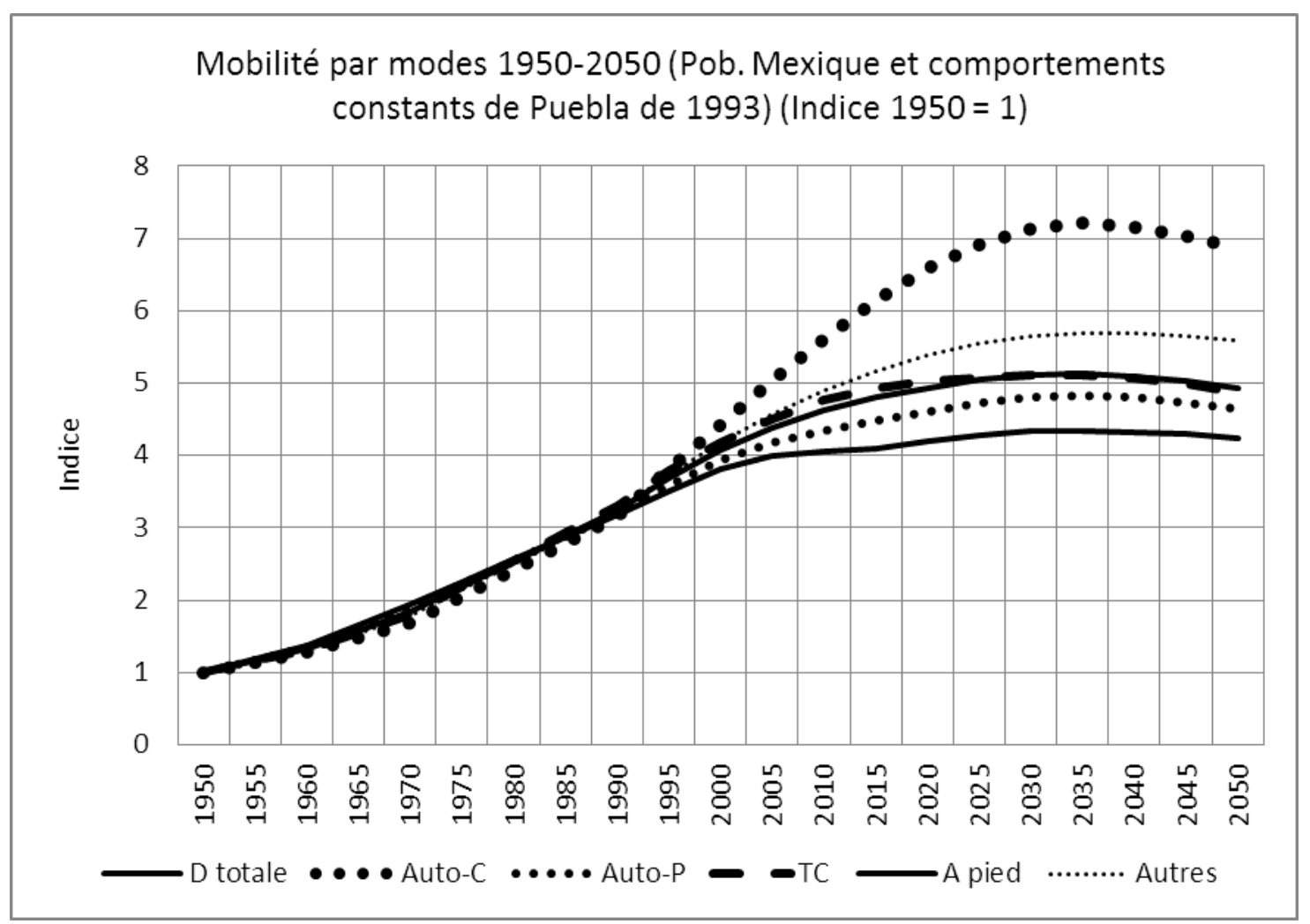

Source : Bussière, oct. 1991. 
Graphique 17: Évolution de la pauvreté (alimentaire, capacités, patrimoine), Mexique, 1992-2008

\section{Evolución de la pobreza por ingresos nacional, 1992 a 2008}

(porcentaje de personas)

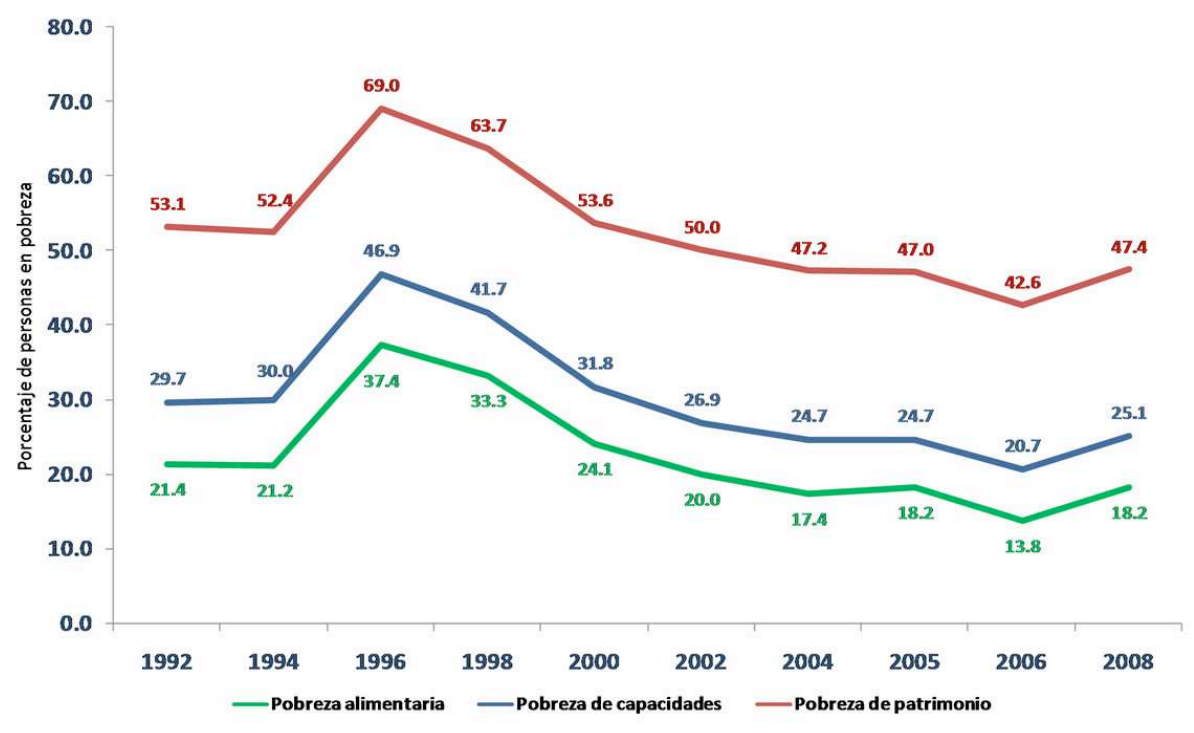

Fuente: estimaciones del CONEVAL con base en las ENIGH de 1992 a 2008

Source: Coneval (2009).

Graphique 18: Simulation sur Puebla - Distance en km par personne tous modes - 19942030

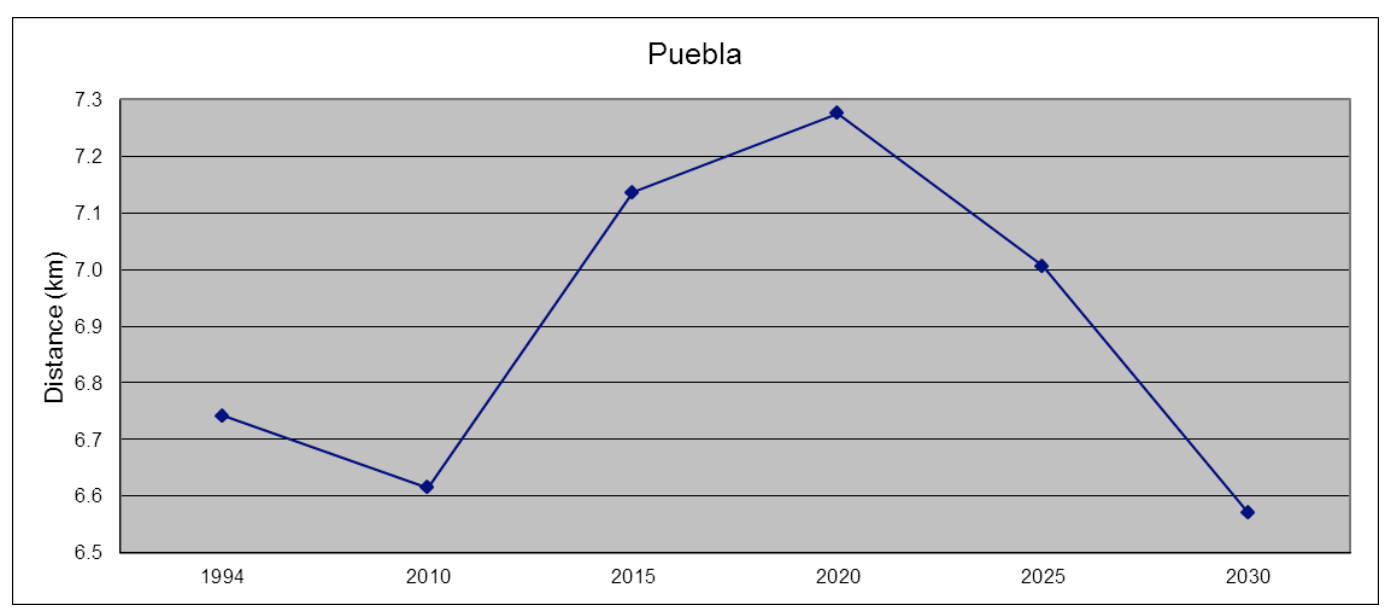


Graphique 19: Simulation sur Ciudad Juarez -- distance en km par personne tous modes - 1996-2030

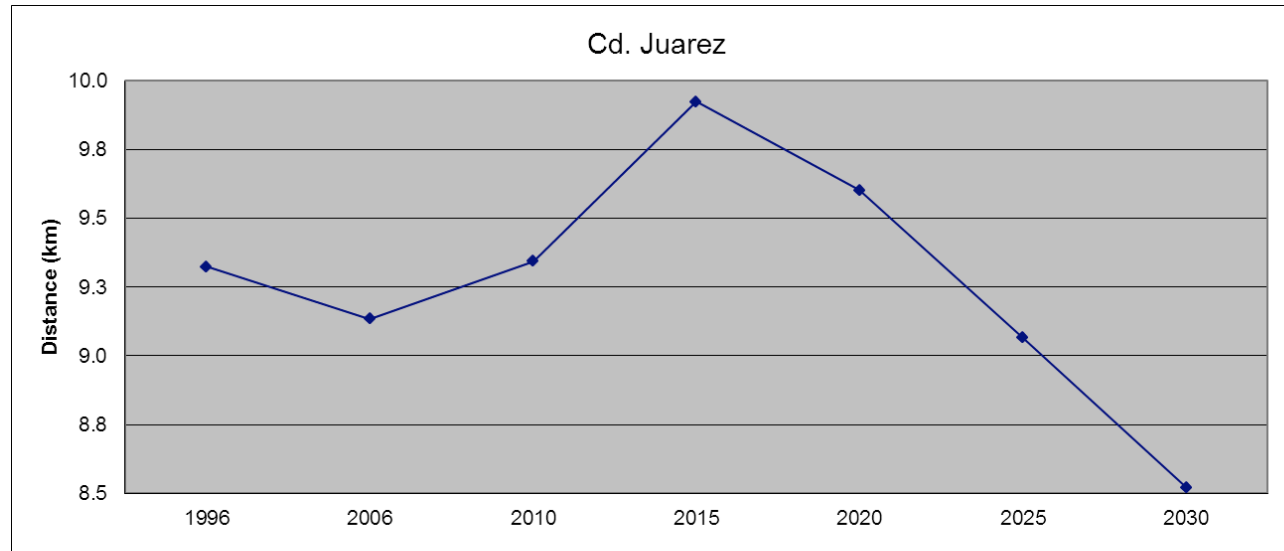

\section{Conclusion}

En résumé pour le cas français, les principaux constats sont les suivants : On y observe la même tendance à la baisse de la mobilité que dans la plupart des pays développés à partir du début des années 2000 alors que le kilométrage moyen par ménage plafonne et baisse légèrement par la suite avec ces variations conjoncturelles liées vraisemblablement aux variations du prix du carburant, baisse du taux de possession du permis de conduire chez les jeunes dans les zones urbanisées les plus denses (ailleurs on observe le contraire), diffusion sociale de l'automobile qui atteint ses limites avec la baisse des inégalités de la possession d'automobile(s) et la hausse généralisée de la $2^{\mathrm{e}}$ auto ; vieillissement du parc automobile, saturation de la possession auto en Île-de-France et ralentissement hors IdF. On observe par ailleurs une progression significative de l'usage des transports collectifs.

Dans nos études de cas au Mexique, nous entrevoyons également un phénomène de saturation qui pourrait se produire dans environ une vingtaine d'années dans les villes plus traditionnelles et un peu plus tôt dans les villes plus développées à condition d'un ralentissement de la croissance démographique. Le parc automobile est cependant vieux et n'a pas tendance au rajeunissement à cause du manque de pouvoir d'achat mais aussi de politiques qui encouragent la propriété de vieux véhicules comme la taxe annuelle sur les véhicules dont sont exemptés les voitures de 10 ans ou plus - taxe qui a été éliminée récemment au niveau national (taxe fédérale) et dans certains états (Puebla et Tlaxcala en 2011).

A partir du constat d'une saturation ou à tout le moins une décélération de la mobilité globale ainsi que de la mobilité automobile urbaine dans les pays développés ainsi qu'à l'apparition probable d'une tendance analogue dans les pays émergents mais seulement dans une vingtaine d'années après de fortes pressions vers une motorisation individuelle, que pouvons-nous conclure en termes de politiques?

Pour les pays développés où la croissance des villes est en train de changer on peut y voir un signe encourageant où il sera plus facile de réorienter la planification du transport urbain: restreindre l'usage de l'automobile en ville en favorisant les transports collectifs et les modes doux; contrôler l'expansion urbaine en densifiant les banlieues et en renforçant les transports collectifs; repenser la construction d'autoroutes à péage en périphérie des agglomérations qui ne sont peut-être plus utiles ni rentables ; revoir notre conception de la qualité de vie en ville moins axée sur la fluidité des déplacements automobile : introduire diverses mesures de gestions de la demande pour diminuer le nombre de déplacements et l'usage de l'automobile en ville. Aussi, agir sur la technologie en introduisant des normes plus strictes aux manufacturiers; cependant l'impact sur le cycle de 
production et le renouvellement du stock pourrait prendre encore une vingtaine d'années. Il faudrait donc accélérer cette transition (Schipper, 2011).

Pour les pays en émergence, malgré les grandes disparités d'un pays à l'autre et d'une ville à l'autre l'exemple de Puebla et de Cd. Juarez peut nous donner une idée de l'ampleur du défi à relever au cours des prochaines années: la croissance démographique sera encore relativement forte pour au moins une génération ou deux, l'expansion des villes se fera d'une façon vraisemblablement désordonnée et étalée, le nivelage socio-économique de ménages en termes de motorisation n'est qu'à ses débuts, les politiques de transport sont pour la grande majorité favorables à l'usage de l'automobile sur le modèle américain des années '70, au moins au Mexique et en Amérique Latine ; les transports collectifs sont pour la grande majorité artisanaux et peu compétitifs face à l'automobile et l'absence d'une fiscalité redistributive rend difficile, hormis dans les très grandes villes, leur modernisation et l'introduction de subventions d'exploitation pour les rendre plus compétitifs ; la faible conscience du public des enjeux environnementaux; les problèmes de sécurité compliquent l'implantation de modes non-motorisés que les villes du Nord adoptent de plus en plus. Quelles seront donc les politiques les plus adéquates?

Premièrement, renforcer les acquis. Beaucoup de villes du Mexique et d'Amérique latine construites sur le modèle européen ont encore des densités élevées comparables aux villes du vieux continent. Élaborer des politiques pour maintenir la densité des villes centres et éviter de construire des boulevards périphériques sans des mesures d'accompagnement pour éviter une hémorragie de population du centre vers la périphérie; moderniser les transports collectifs pour les rendre plus compétitifs face à l'automobile et modifier leur image d'un transport pour pauvres à un transport pour tout le monde ; favoriser l'implantation de zone piétonnières dans les villes centre et dans les banlieues; favoriser le maintien de l'usage de la bicyclette dans de nombreuses villes où il n'a pas encore disparu; promouvoir l'expansion de l'usage de la bicyclette utilitaire et récréative; réglementer pour rajeunir le parc automobile avec des véhicules plus propres ; diffuser l'information et faciliter les procédures pour avoir accès aux bons de carbone qui pourraient financer ces mesures.

Oui, la tendance à toujours plus de mobilité urbaine, qui semble s'inverser dans les pays développés, devrait se généraliser à plusieurs pays émergents mais seulement dans une vingtaine d'années. Les défis à relever pour un transport soutenable demeurent entiers.

\section{Remerciements :}

Nous remercions le Cuerpo Académico de Economía Urbana y Regional de la Faculté d'Économie de la Benemérita Universidad Autónoma de Puebla ainsi que le CONACYT du Mexique (subvention CB-2011-01 no. 168388) pour leur appui.

\section{RÉFÉRENCES}

ADEUS (Agence de développement et d'urbanisme de l'agglomération strasbourgeoise) (2010). Baisse de la mobilité et modes de vie en évolution, Les notes de l'adeus, mars.

Avrillier, P., Hivert, L., Kramarz, F. (2010). « Driven Out of Employment? The Impact of the Abolition of National Service on Driving Schools and Aspiring Drivers », 24 p., first published online: 17 Aug. 2010, Online ISSN: 1467-8543, publié dans British Journal of Industrial Relations, Volume 48, Issue 4, december 2010, pp784-807, C Blackwell Publishing Ltd/London School of Economics.

BITRE (Bureau of Infrastructure, Transport and Regional Economics) (2012). "Traffic Growth: Modelling a Global Phenomenon", Report 128, Canberra ACT.

Bussière, Y. D. (oct 2011). Paris (Marne-la-Vallée). Conférencier invité. « Puebla : Évolution de la mobilité 1994-2011 ». IFSTARR, DEST. 27 oct., Ppt, 54p.

Bussière, Y. et L. Fortin (1990). «Demande de transport et évolution démographique : rétroprojection et prospective $»$. Communication, $14^{\mathrm{e}}$ congrès annuel de l'Association canadienne 
des sciences régionales, Univ. de Victoria, Victoria, Canada, 2-4 juin. 26p.

Bussière, Y., Madre, J.-L., Armoogum, J., (1996). "Vers la saturation ? une approche démographique de l'équipement des ménages en automobile dans trois régions urbaines", Population, $\mathrm{n}^{\circ}$ 4/5, pp. 955-977.

CCTN (Commission des Comptes Transport de la Nation) (2012). Ministère de l'Écologie, du Développement durable, des Transports et du Logement. Les comptes des transports de la Nation en 2011. Juillet.

Collet, Roger. (2012). Household car use in France : a demographic and economic analysis. Economics Bulletin, 2012, vol. 32, issue 1, pp. 475-485.

Coneval - Consejo Nacional de Evaluación de la Política de Desarrollo Social (2009). Evolución de la pobreza en México. 24p. pdf. www.

Davis, B., Dutzik,T., Baxandall, Ph. (2012). "Transportation and the new generation: Why Young People AreDriving Less and What It Means for Transportation Policy ?" Frontier GroupU.S. PIRG Education Fund.

Dupuy G. (1999). "La dépendance automobile : symptômes, analyses, diagnostic, traitements", Paris, Anthropos.

Gardes F., Langlois S. et Richaudeau D. (1996). "Cross-section versus time-series income elasticities", Economics Letters, 51, p. 169-175.

Goodwin, Ph., (2010-2011). "Peak Car. A series of five articles in Local Transport Today, June 2010-June2011" Local Transport Today, London, 14p.

Hivert, L., (Nov. 2011). "Short-term break in the French love for diesel ? ", accepté dans Energy Policy, Corrected Proof in press, disponible en ligne 25/11/2011, à paraître dans le numéro special «Special Issue : Decades of Diesel » (2012?).

Hubert, J.-P., (2009). « Dans les grandes agglomérations, la mobilité quotidienne des habitants diminue, et elle augmente ailleurs », INSEE Première $n^{\circ} 1252$.

Hubert, J.-P., Madre J.-L., Roux S. (2010). "Changes in Fractioning Behaviour on Work Days", IATUR (International Association for Time Use Research), Paris.

Kolli, Zéhir. (2012). Dynamique de renouvellement du parc automobile. Projection et impact environnemental. Thèse de doctorat en Sc. Économiques, Univ. Paris 1. Panthéon-Sorbonne, 309p.

Kuhnimhohf, T., Armoogum, J., Buehler, R., Dargay, J., Denstadli, J.M., Yamamoto, T., (2012). "Trends in Young Adults'Behaviour: Evidence from Six Industrialized Countries",90th TRB Annual Meeting, IFSTTAR, Document de travail.

Litman, T. (2011). The Future Isn't What It Used To Be. Changing Trends and Their Implications For Transport Planning. Victoria Transport Policy Institute. 49p. www.vtpi.org/future.pdf .

Madre, J.-L., Armoogum, J. (1997). "Interview et présence au domicile" XIV Symposium annuel international sur les questions de méthodologie, Statistique Canada, Ottawa (publication d'une version condensée en français et en anglais).

Meissonnier J., (2011). "Le déménagement: un déclencheur des changements dans les choix modaux ? Le cas de trois agglomérations du nord de la France", Dixièmes rencontres francophones Est-Ouest de socio-économie des transports, DRI - Ecole polytechnique de Montréal, 1-5 juin 2011, Montréal, Canada.

Metz. D. (2010). Saturation of Demand for Daily Travel, Transport Reviews, 30: 5, 659-674.

Millard-Ball, A., Schipper, L. (2010). "Are We Reaching Peak Travel ? Trends in Passenger Transport in Eight Industrialized Countries", Transport Reviews. http://www.tandfonline.com/doi/abs/10.1080/01441647.2010.518291.

Newman, P. and J. Kenworthy (2011). " 'Peak Car Use': Understanding the Demise of Automobile Dependence", World Transport, Policy \& Practice, vol. 17.2, June. Pdf 14p. www.

Noble, B. (2005). Why are some young people choosing not to drive ? Proceedings of the European Transport Conference. Strasbourg.

OECD/ITF, International Transport Forum, (2011). Peak car travel in advanced economies?, 
Chapter 3, Transport Outlook: Meeting the Needs of 9 Billion People, Paris.

Papon F. et Hivert L. (2008). "Adulterous behaviour within the car-owner couple: some analyses from French panel data on car rental and car sharing within households", IATSS Research, 32(2), p. 6-15.

Quételard (2011). "Une rupture dans l'évolution de la mobilité urbaine : Les enseignements des dernières EMD", Atelier thématique RST "Demande de Transport : méthodes de recueil, analyses et prospective", Certu, 6 décembre.

Richards F.J. (1979). "A flexible growth function for empirical use". Journal of Experimental Botany, 10, pp. 290-300.

Roux S. (2012). "Transition de la motorisation en France au XXème siècle", Doctorat en Démographie, Paris I - Panthéon - Sorbonne.

Schipper Lee (2011). "Automobile use, fuel economy and CO2 emissions in industrialized countries : Encouraging trends through 2008 ? », Transport Policy 18, 358-372.

Sivak M, Schoettle B. (2012) "Recent changes in the age composition of drivers in 15 countries", Traffic Injury Preention, 13:126-132.

SOeS (2012). "La circulation routière augmente à un rythme ralenti depuis 2003 ", Le Point sur $\mathrm{n}^{\circ} 118$, CGDD. www.statistiques.developpement-durable.gouv.fr TRANSPORT

STIF (2012). "Nouvelles mobilités des franciliens", http://www.stif.info/IMG/pdf/Enquête_globale_web.pdf -

http://www.stif.info/IMG/pdf/Nouvelles_mobilites_des_franciliens_9_juillet2012.pdf. 